\title{
Oscula mariniana
}

Presento qui una campionatura di diciannove commenti - tanti sono i baci del Marino nel gruppo tematico più vistoso della seconda parte della Lira destinati all'edizione di Madriali e canzoni che, insieme ad Alessandro Martini, conto di portare a termine fra breve. Ė inutile ridire dell'opportunità di un lavoro del genere oggi che i nuovi studi secenteschi hanno da più parti indicato la necessità di scavi e recuperi filologico-testuali. Mi limito a ricordare brevemente alcuni elementi distintivi dell'intera Lira che possono servire da supporto immediato, soprattutto a chi non frequenta assiduamente i luoghi del Cavaliere, per una lettura ravvicinata dei suoi prodigi retorici.

Il "rustico, ma nuovo ordine" del canzoniere dichiarato dal poeta nella dedicatoria delle Rime (1602) a Melchiorre Crescenzi si esprime nella prima parte dell'intera raccolta con l'abbandono dell'impianto biografico (o pseudobiografico) e con una partizione della materia poetica in otto sezioni; le quali saranno ridotte a quattro nella terza parte (1614), dove il titolo, Lira, si intende esteso alle due precedenti. Nella seconda parte, già Rime II, Madriali e canzoni, non c'è segmentazione tipografica e titolazione delle diverse sezioni; ma queste sono comunque riconoscibili in tre grossi blocchi, corrispondenti a liriche di argomento amoroso (più della metà dei 225 componimenti della raccolta), figurativo (che per la maggior parte confluiranno nella Galeria, del 1619) e sacro. All'interno di questi blocchi il Marino raggruppa altri nuclei tematici più specifici, altre sezioni di lunghezza variabile, di solito intervallate dai componimenti più lunghi. Una di queste è appunto quella dei baci che si estende da 13 a 31.

11 tema fu particolarmente caro al poeta, come attestano numerose altre occorrenze nel vasto corpus della sua produzione lirica e nell'Adone. Qui egli lo affronta per la prima volta e in maniera già sistematica e organica: non solo con la quantità dei componimenti e con richiami diretti a precedenti cultori del tema del bacio (Secondo, Tasso, Guarini e Rinaldi soprattutto), ma anche con messaggi extraverbali affidati alla dispositio dell'intera sezione. E se è poi vero che nella poetica del Marino manca una precisa linea di sviluppo, almeno per quel che riguarda la trattazione dei temi prediletti, sarà questo un luogo privilegiato di riscontri utili per l'intera sua opera (ed egli stesso, del resto, vi 
Secondo: un'operazione che sarà ripetuta, come ha notato nel suo commento Giovanni Pozzi, con diciannove ottave nel canto 8 del poema grande (410). Prima di considerare le implicazioni di questo voluto collegamento, bisogna fermarsi un attimo per certificare la misura della sezione in quanto il titolo dell'ultimo pezzo (31, Amori notturni) sembrerebbe indicarne l'estraneità ad essa. Una prima prova dell'integrità che qui si afferma, visto che non ci sono problemi a far iniziare la sezione con $\mathbf{1 3}$, è offerta dal netto distacco tematico tra 31 e 32, una seconda dalla linearità del filo narrativo tra 13 e 31 . Nel primo caso abbiamo un triplice amplesso notturno annunciato da spessi, lunghi, e molli baci (31.34-35), logica conclusione della precedente Guerra di baci (30), cui si oppone un luminoso quadro primaverile (32) che è giusto prologo di una sezione completamente nuova e diversa, di natura, per così dire, "boschereccia". Nel secondo caso abbiamo una 'storia' con i suoi ben definiti punti di inizio e fine. Si tratta, per la verità, di una storia doppia o, meglio, iterata. Si può partire, per intenderla, da dove presumibilmente partì il poeta stesso nel disporre in "nuovo ordine" (tematico essenzialmente, ma poi decisamente narrativo all'interno delle sezioni e sottosezioni) le rime osculatorie composte sicuramente in occasioni diverse, come certifica 1' 'anzianità' della Canzone dei baci. Si può partire dal desiderio di emulare Giovanni Secondo, e cioè dal numero diciannove. Che tante e non più o meno dovessero essere le tessere della sezione è assicurato, inoltre, dal fatto che altri componimenti di B, pure osculatorî (come il bacio del vecchio sdentato, 38, e i mille baci dei Numeri amorosi, 50, per non dire di 118, 147, 208) non sono inseriti qui, ma collocati in altre sezioni. C'è poi la Canzone dei baci cui per motivi almeno sentimentali andava assegnata una posizione di rilievo. Ora tale canzone, tematicamente e narrativamente, va dal bacio all'amplesso, e con tanto di eiaculazione, come si vedrà. Marino vuole raccontare proprio questa storia, ma espansa: dal bacio furtivo, e per giunta solo desiderato (13), all'orgasmo multiplo (31). Si potevano, certo, allineare le diciannove tessere in maniera progressiva, assegnando quindi le due ultime posizioni a 20 e 31; ma il poeta, lo sappiamo, giocava sul doppio: l'attrazione per il modulo binario lo portava verso una soluzione iterativa o chiastica. Prevalse quella iterativa perché essa offre al tema maggiori possibilità espressive: il chiasmo è segno di immobilità, mentre il bacio per Marino è dinamico, nel senso che 'porta' all'amplesso.

Il numero dispari da cui deriva la dispositio della sezione individua un centro che cade in 22, Baciator dubbioso. Il dubbio costituisce una pausa narrativa, un momento di ristagno emotivo dopo che $\mathrm{i}$ primi baci hanno condotto al singolo appagamento sessuale descritto nella prima canzone, 20 , e alla successiva separazione da cui nasce la nostalgia di $\mathbf{2 1}$, Tornate o cari baci. Al dubbio del madrigale centrale, 22, è legata la riflessione del poeta intorno alla natura del bacio con la distinzione tra i sensi (vista e tatto) coinvolti nell'esperienza osculatoria; l'argomento ritornerà in 26 e nello spazio più 
disteso delle canzoni 27 e 28 quando comunque il 'movimento' che condurrà al nuovo amplesso, ovvero lo sviluppo narrativo, sarà già pienamente avviato. Nella sosta di 22 si dichiara la doppia natura del bacio con una serie vistosa di elementi formali e tematici disposti a due a due. E il 'doppio' che dunque identifica il centro, da cui si possono 'vedere' i due segmenti dello stesso numero di componimenti che 'raccontano' la stessa storia sviluppata progressivamente dal bacio all'amplesso. C'è però una variante di rilievo: nel primo caso l'iniziativa parte dal desiderio del poeta (13), mentre nel secondo da quello della donna (Eccomi pronta ai baci, 23). Pare legittimo pensare a un erotopaegnion: i due segmenti dell'intera sezione 'si baciano' come il poeta e la sua donna che appunto, alternativamente, chiedono e ottengono baci. $\dot{E}$ il poeta stesso che sembra suggerirci questa figurazione ricordandoci che il bacio vien doppio (20.48) e mostrandoci come lo stimolo possa partire sia dalla donna (Bacianne, Aminta mio / io bacio se tu baci / bacia, ch'io bacio anch 'io, 27.8-11) che dall'uomo (Raddoppiam dunque i nodi / cara mia Clori amata, 27.33-34).

L'iterazione è una figura di collocazione che rispetto al chiasmo, abbiamo ricordato, ha un certo dinamismo in quanto espressivamente accumula e lancia in avanti. Troviamo, infatti, che la storia è ripetuta con una notevole intensificazione: la prima volta si conclude con un solo amplesso appagato (20.85-87), la seconda con due $(31.42-44 ; 31.78-83)$. Una progressione è mostrata anche nel ricorso al 'silenzio', da quando il poeta lo raccomanda a sé stesso dopo il primo bacio, rubato (19), fino a quando si trasformerà drammaticamente in oblio eterno dopo l'ultimo amplesso (31.125). L'originalità del Marino rispetto ai numerosi autori imitati, tradotti o anche soltanto ricordati, crediamo sia da ricercare soprattutto in questa insoddisfazione crescente e, alla fine, totale. Narrativamente è motivata in $\mathbf{3 1}$ dall'irritazione per la mancata terza erezione, ma essa è molto più che un imbarazzante, e vuoi pure comico, accidente notturno. Il condurre a logica conclusione sessuale i mille sospiri petrarcheschi e petrarchisti non dà al poeta la tanto agognata pace dei sensi e dell'anima. Al contrario, l'appagamento del desiderio gli spalanca dinanzi la visione dell'abisso: un vuoto esistenziale angoscioso che non si può evitare gingillandosi con la vaga attesa del possesso della donna, da precedenti poeti sempre rimandato con compiacimenti manieristici e senza alcun turbamento. Qui siamo di fronte a eccessi sconvolgenti e perfino alienanti: quando l'appagamento sessuale si è realizzato e la figura della donna è stata svuotata da ogni simbolismo religioso o anche semplicemente spirituale, il poeta può solo reagire ingaggiando una lotta disperata con il destino avverso: tentando di riprodurre i momenti dell'estasi e di annullarsi nel piacere osculatorio (trasformar mi vorrei tutto in un bacio, 28.30), oppure di consumarsi nell'amplesso (stanco, non sazio..., 31.89). Questo ultimo e disforico approdo dei baci può dunque intendersi come il tratto poetico distintivo del Marino in materia amorosa (se percepito qui, beninteso, nell'arco dell'intera doppia 
vicenda narrata, ché a voler prendere ogni componimento separatamente difficile sarebbe riconoscerlo).

La programmaticità di questa ideale 'dichiarazione' mariniana intorno all'amore, sebbene sia tutta a posteriori, ovvero espressa essenzialmente nella dispositio, distingue questa esperienza poetica sia da quelle parallele del Guarini, del Rinaldi, del Casoni e di altri (si veda la ricchissima sezione osculatoria del Gareggiamento ${ }^{2}$ ) che non risultano teoricamente impegnate, e sia da quella precedente di Giovanni Secondo. Al quale il Marino volle riconoscere probabilmente un merito trattatistico-enciclopedico proprio per mostrarne il superamento con il suo nuovo e pregnante catalogo. L'umanesimo latino tardo e settentrionale del Secondo non poteva certo competere con le raffinate pulcritudini manieristiche dei poeti italiani di fine secolo; ma nessuno di questi, d'altra parte, aveva elaborato sistematicamente il tema. Con mossa anfibologica il Marino attinge dall'una e dall'altra parte nello stendere i suoi baci e nel dare ad essi, e cioè alla propria espressione poetica del bacio, una moderna e coerente dimensione.

\section{3 - Mad. 13 - Desiderio di bacio furtivo}

1 Di furto Amor nascesti $\mathrm{e}$ ' $\mathrm{n}$ virtù d'un bel volto di furto il cor m'hai tolto. E'l bel volto ch'adoro,

5 quando formò natura nevi e perle, ostro e oro quinci e quindi togliendo e gigli e rose, pur di furto compose. Or consenti che tolga al'idol mio 10 di furto un bacio anch'io.

Il paralogismo del madrigale, fondato sulla convenzione del furto d'amore, è espresso sintatticamente in tre diversi momenti, riconducibili comunque a due secondo lo schema retorico precedentemente delineato. Invocando Cupido, nell' 'enunciazione' (1-8) il poeta allinea tre esempi che chiariscono la normalità, o addirittura la legge, del furto amoroso. Nella 'riflessione' (9-10) egli chiede il giusto consenso del dio prima di mettere in pratica il suo proposito. Non risulta alcuna versione musicale di questo componimento, probabilmente a causa dell'ossessiva ricorrenza di furto che porta l'accento tonico sulla vocale meno cantabile; altre motivazioni potrebbero essere la presenza di due rime irrelate (di cui la prima in -esti senza riscontri allitterativi o paronomastici) e la musicalmente eccessiva estensione del secondo periodo (4-8).

La paternità di Cupido è qui assegnata a Marte per effetto degli amori 
"furtivi" consumati con Venere (narrati da Momo nell'Adone [7.167-228]), mentre altrove (114, Dal zoppo genitore) a Vulcano. La stessa alternanza si registra anche, e in posizioni più prossime, nel poema grande (Marte: 1.9; Vulcano: 1.67), segno che il poeta non si fa scrupolo di appellarsi a diverse genealogie per esigenze immediate di composizione.

Il furto osculatorio non compare nel pur ricco campionario di Giovanni Secondo, ma sovrabbonda tra i poeti più vicini al Marino, al punto che un'intera partizione del Gareggiamento è intestata al Bacio involato (53r-55r). In essa compaiono madrigali di Tasso, Guarini, Rinaldi, Leoni, Petracci, Contarini, Moro e Scaglia, insieme, naturalmente, ad alcuni pezzi del Marino stesso. Tra i baci del Gareggiamento i più prossimi a questo del Nostro appaiono Qual'hor furtivo la mia donna bacio dello Scaglia, il quale ricorda che Amore nacque ancor di furto e di rapina, e $O$ che soave bacio del Guarini, in cui troviamo fatti pur ladro Amor, Amor, ch'io ti perdono. Non si può escludere, infine, un ricordo delle rime 305 e 306 del Tasso.

Baci rubati ricorrono anche altrove nell'opera del Marino. Se ne trovano in Bo 36, CAm 81.71-74 e, numerosi, nell'Adone (3.152; 12.243; 13.189; $14.296 ; 15.103 ; 16.57 ; 18.187 ; 19.264)$. È comunque in questa sezione di B che essi appaiono più compatti, e tutti $(13,14,18,19)$ nel primo dei due segmenti che la compongono, e cioè nella prima 'storia' (13-21), perché nella seconda la donna è pronta ai baci e non c'è più bisogno di rapine.

14 - Mad. 14 - Bacio bramato

1 Un bacio, un bacio solo,

Filli, il doni o l'involo?

Se 'l doni, e' fia gradito, che dolce bacio è quel che porge e scocca

5 il cor più che la bocca.

Se "l furo, amante ardito,

fia dolce ancor, ché non men dolci sono

furto i baci che dono.

Un sol bacio, un sol bacio,

10 o rapito, o donato, far non mi può giamai senon beato.

La collocazione di questo madrigale si spiega facendo riferimento allo sviluppo della vicenda osculatoria così come è descritta dagli "argomenti". Da precedente desiderio (13) si passa qui alla brama. Dopo la quale il poeta, rivoltosi finalmente alla donna in modo diretto, apre la fase triplice della 'richiesta' (15, 16, 17). Questa, evidentemente respinta, conduce prima al "furto" (18), quindi alla soddisfazione che ne risulta (19), e infine alla totale reciprocità osculatoria espressa nella Canzone dei baci (20). 11 crescendo è anche assicurato dal tópos 
del bacio come promessa di ulteriori piaceri, già presente in Secondo (n. 3) e confermato da un gruppo di madrigali del Gareggiamento (57r-58r) che, sotto l'intestazione Un bacio è poco, è aperto da un eloquente bacio del Guarini, ...segno / di futuro diletto.

La struttura del componimento, che consta di quattro periodi, è giocata sul contrasto tra l'unità del bacio (un bacio solo) e la doppia possibilità di realizzarlo (il dono o l'involo). Il modulo doppio prevale come sempre: sintatticamente è assicurato dal gemellaggio dei due periodi centrali e dal raccordo del quarto (un bacio, un sol bacio...) con il primo (un bacio, un bacio solo...). La binarietà prevale sull'unità anche all'interno dei singoli versi; ognuno di essi, infatti, si impernia su una coppia di lemmi, associati per identità, somiglianza, contiguità semantica, o per opposizione: 1. bacio bacio; doni - involo; 3. doni - gradito; 4. porge e scocca; 5. cor - bocca; 6. furo - ardito; 7. dolce - dolci; 8. furto - dono; 9. bacio - bacio; 10. rapito - donato. Il segno del doppio nell'ultimo verso, infine, si riscontra nella doppia negazione, non. Una ulteriore conferma di questa insistita binarietà viene dal sistema delle rime: cinque a coppie e una irrelata; la quale, comunque, è portata da un verso composto dal raddoppio del sintagma un sol bacio.

Nell'ambito dell'intertesto mariniano si deve innanzi tutto ricordare l'iterazione di un bacio solo in Bo 36 (2-3), seguita nello stesso componimento da io moro (9) richiamato due volte nell'incipit di 15. Nell'Adone non si offrono baci ostentatamente solitari come questo, ma vi compare un sicuro aggancio ai versi 3-5 nel celebre detto di Venere: Amor del bacio è fabro, / il cor lo stilla, il labro poi lo scocca, / il più ne gode l'alma, il men la bocca (8.125), che è poi un ritocco del qui prossimo Amor del bacio è fabro, / egli il forma, ei lo scocca; / pria dal cor che dal labro / dolcemente trabocca, / ma' $l$ sente e gode il cor più che la bocca (28.41-5).

Per quanto riguarda i riferimenti ad altri autori, ancora una volta è il Gareggiamento che offre le schede più sicure: da Un bacio solo un bacio di Maurizio Moro (57v) a Tu mi dici ch'io chieda di Francesco Contarini (58r), in cui troviamo che il bacio o il dona o me l'involo, con conseguente doppio sviluppo (se il doni tu... s'io l'involo...) come in Marino. Dono e rapina si trovano insieme anche in Rinaldi, che così conclude un madrigale: Poiché Amor ti destina / al dono, a la rapina, / io dono, invola tu, né dir t'aggrave, / se 'l donar, se 'l rapir sia più soave (Delle Rime. Parte terza 8).

Il Nuovo Vogel riporta tre versioni musicali del componimento, nn. 624, 205 e 1266, rispettivamente del 1607, 1611 e 1646.

15 - Mad. 15 - Bacio chiesto con arguzia

1 lo moro, ecco ch'io moro: 
bella nemica mia t'offesi assai, levar tropp'alto i miei pensieri osai.

Perdon ti cheggio, in pegno

bramo di pace un segno:

in questa estrema mia dura partita

non vò senza il tuo bacio uscir di vita.

La microvicenda del bacio chiesto si articola in tre momenti definiti da questo e dai due madrigali successivi. Narrativamente la richiesta vera e propria è effettuata qui nel primo componimento, mentre nel secondo troviamo la reazione negativa della donna, e nel terzo la 'scherzosa' fantasia del poeta intorno a una possibile, ma negata, entusiastica accettazione. Gli argomenti, qui come altrove spie veraci di autoesegesi mariniana, oscillando tra arguzia e scherzo, indicano una certa leggerezza manieristica, disimpegnata sul piano dei contenuti, che collega direttamente alle esperienze poetiche dei rimatori contemporanei. Tra i quali, infatti, il tópos del bacio associato alla morte 'scherzosa' è piuttosto frequente; le sue manifestazioni madrigalesche possono essere di due tipi, qui fusi dall'arguzia mariniana: da una parte sta il bacio che uccide o comunque avvicina alla morte, e dall'altra sta il bacio che ridà la vita (tralasciamo per ora sviluppi più densi in cui esplicitamente, come si avrà modo di osservare nella Canzone dei baci, quel morire è vita, 20,98).

Tra i sempre ovvi riferimenti del Gareggiamento si ricordano, per il primo gruppo, la chiusa di Morrò per voi mio sole di Agostino Nardi (a che non m'ancidete / col tosco omai che su le labbra avete [49v]), e quella di Non avventar più strali del Rinaldi (Baciami una sol volta, / e col bacio mi fia la vita tolta [48v]). Entrambi i madrigali compaiono accanto a questo del Marino tra i Baci chiesti della raccolta, e ad essi va aggiunto il bacio ricevuto del Conte Marco Verità: Baciava avido Elpino / ... / trovar pensando a' suoi tormenti aita / e ne perdé la vita (61r). Per il secondo gruppo, si ricordano ancora il Rinaldi (Corre al suo fin mia vita, / né fuor ch'un bacio attende / un bacio ohimé, che sol può darle aita [57r]) e Maurizio Moro (... ma ritrosa lo neghi / perché con poca aita / ancor ricusi di tornarmi in vita [57v]).

Per l'accostamento topico del bacio a morte e vita si possono raccogliere numerosissimi altri riferimenti cronologicamente più distanti, tra cui notevoli paiono questo bacio in punto di morte del Secondo,

Iam Styx ante oculos et regna carentia sole,

Luridaque annosi cymba Charontis erat,

Cum tu suavolium educens pulmonis ab imo

Adflasti siccis inriguum labiis,

Suavolium, Stygia quod me de valle reduxit

Et iussit vacua currere navem senem. (Basium n.13)

e questi negati o secchi e curti dell'Ariosto, 
ma quelli baci ancora, a' quai risurti miei vital spirti son spesso da morte, mi niega o mi dà a forza secchi e curti. (Rime 70.67-69)

Nell'intertesto mariniano s'avranno infine da ricordare la piccola arguzia osculatoria di Ma 17 (offerta di coralli in cambio di coralli-baci); Bo 17: O più crudel che gli avvoltori e i nibi / pasciti del mio cuore e del mio sangue, / pur ch'un tuo bacio anzi 'l morir delibi (T2); e soprattutto Bo 36: Io moro e giunta omai presso l'uscita / l'anima è già, se tu presta non sei / con la tua bocca a ritenerla in vita (T1).

Questo è il madrigale più musicato di tutta la sezione dei baci. Il Nuovo Vogel ne indica ben ventidue partiture, di cui tre (di Pomponio Nenna, Tommaso Pecci e Salomone Rossi) dell'anno 1603. Evidentemente si tratta di un componimento che risponde perfettamente alle esigenze testuali dei musicisti, i quali privilegiano "la percezione delle qualità sonore prima ancora che logiche" (Bianconi 322-23) e mostrano una acuta "predilezione per lo stile medio" (336). Le rime sono facili e quindi cantabili, tutte baciate; anche la prima, a ben vedere, in quanto portata da un verso in cui il termine semanticamente più significativo, moro, è ripetuto, e segnato in entrambi i casi, nella stessa posizione sillabica, da ictus primario. Offrono sonorità musicalmente apprezzabili anche varie assonanze (nemica - mia, 2; cheggio - pegno, 4), le vocali della seconda rima (-ai) che ritornano invertite nella quarta $(-i a)$, le frequenti paronomasie (nemica mia, t'offesi assai, 2; pensieri osai, 3; estrema mia, dura partita, 6).

16 - Mad. 16 - Bacio chiesto

1 Perch'un bacio chegg'io mordi il dito e minacci, bocca spietata, anzi, m'ingiuri e scacci? Sì ch'un bacio desio!

5 Baciami, e poiben mio mordi, minaccia, ingiuria pur, se sai; ché non saranno allor, benché mordaci, minacce, ingiurie e morsi, altro che baci.

Il bacio chiesto è subito rifiutato. Ma intanto si è stabilita una comunic-azione. La donna che ricordiamo di marmo (12) ha per la prima volta risposto al poeta. 11 rapporto si instaura su un piano di conflittualità, cui non manca una certa dose di violenza, che fa da prologo alla rapina osculatoria denunciata in 18 . Il lessico è dominato dalla triplice presenza di tre termini duri, 'petrosi'. Ma con calcolata distinzione: nella parte enunciativa del madrigale (1-4), che si riferisce alla 
reazione della donna, troviamo bocca spietata e scacci accanto alla triade di base, mordi, minacci, ingiuri; solo questa è ripresa, e per due volte, nella conclusione riflessiva (5-8) ed euforica del poeta, che dunque esorcizza la feroce ritrosia della donna normalizzandola nella desiderata esperienza, collaudata dalla tradizione, del bacio mordace. Che ad essa si tenda è assicurato, oltre che dal breve inciso del verso 7 , dalla presenza quadrupla, e dunque prevalente, del termine mordere; che concorre anche a dare regolarità e varietà all'ordine della triade di cui fa parte. Abbiamo infatti:

$\begin{array}{lll}\text { mordi } & \text { minacci } & \text { ingiuri } \\ \text { mordi } & \text { minaccia } & \text { ingiuria } \\ \text { mordaci } & \text { minacce } & \text { ingiurie }\end{array}$

morsi

in cui comunque il mordaci della concessiva (7) sta a sé ritmicamente, favorendo (a voler giustamente evidenziare il termine) l'insorgere di un chiasmo conclusivo che esprime appunto la 'mordacità' dei baci desiderati:

mordi

minacce ingiurie minaccia ingiuria

morsi.

Anche sul piano degli effetti fonici il componimento è giocato sull'iterazione e sul chiasmo. L'unica rima irrelata, C $(-s a i, 6)$ è assonanzata sia rispetto a bB (-acci, 2-3) che a DD (-aci, 7-8), ma portata da vocali in rapporto chiastico rispetto a quelle finali di due termini dello stesso verso (minaccia, ingiuria). Ancora: all'iterazione vocalica di bacio e chegg'io (1), seguono il chiasmo di mordi (2) e il ribaltamento successivo di dito (2), così come a quella di bacio e desio (4) seguono poi e mio (5). E infine, con l'allitterazione 'mordente' della " $r$ ", prevalentemente sul raddoppio e sullo scambio delle vocali "u" e "o" risultano posizionate le paronomasie degli ultimi versi: mordi, ingiuria, pur (6); saranno, allor mordaci (7); ingiurie, morsi (8).

Sul tema del bacio mordace più si dirà nel commento al madrigale ad esso intestato (23) che a questo si richiama perché vi si realizza l'identità morsobacio qui espressa soltanto dal desiderio. Conviene però ricordare subito il seguente, per la verità poco felice, Bacio chiesto di Valerio Belli, che direttamente rimanda sia all'inizio del madrigale mariniano (il morso di stizza della donna), che alla sua fine (il piacere del bacio):

Non ti morder le labbia cara vita mia bella, 
che la mia non la tua lingua è rubella;

essa "1 disse da rabbia [,]

amorosa puniscilla; ma pria

ne la tua bocca prigioniera stia. (Gareggiamento 48v)

Il Nuovo Vogel elenca quattro raccolte musicali che includono il componimento mariniano, una del 1621 e tre del 1628.

17 - Mad. 17- Scherzo di bacio chiesto

1 Muto stato fuss'io

quando un bacio ti chiesi,

picciol ristoro a' miei desiri accesi.

Ma, se muto non fui,

5 m'avestu sdegnosetta

(o del mio folle ardir degna vendetta)

co' propri labri, anzi co' denti tui

turata allor la bocca,

svelta la lingua temeraria e sciocca?

È degna di nota la leggerezza che, con lo scherzo qui e con l'arguzia in 18, il poeta assegna a questi madrigali posti in prossimità della conclusione (20) della prima vicenda osculatoria; soprattutto se la si accosta alla 'gravità' degli argomenti di 29 e 30, Baci dolci amari e Guerra di baci, che precedono il secondo e multiplo amplesso di 31 . Le indicazioni del poeta puntano, nel crescendo dell'iterata vicenda, verso un progressivo ma contenuto strani-amento che dal 'tacere' porta all' 'oblio' come si annunciava nel commento iniziale all'intera sezione. Nella chiusa di questo scherzo (turata la bocca, 8; svelta la lingua, 9) già si annuncia il taci, sempre conclusivo, di 19 e 20.

Il madrigale presenta una struttura sintattica di inconsueta complessità, che è forse la prima ragione del mancato interesse dei musicisti; non ne risulta, infatti, alcuna partitura nel Nuovo Vogel. L'enunciazione iniziale (1-3), una proposizione ottativa, non presenta anomalie. Ma la riflessione sul tema (4-9) è costituita da una falsa ipotetica in cui la protasi è aperta da congiunzione avversativa (4), e l'apodosi, con soggetto diverso, si presenta come una seconda e doppia ottativa ( $m$ 'avestu [m'avessi tu] turata... la bocca e svelta la lingua) all'interno della quale è contenuta una terza esclamazione (6).

La prima rima -io, irrelata, offre più all'occhio che all'orecchio nell'essere ripresa non accentuata nel mezzo del secondo verso (bacio). Un altro ostacolo per il musicista è la doppia rima in -ui, per l'inevitabile rilievo da assegnare alla vocale "u", che appare anche in muto (due volte, ed entrambe con ictus primario) e quindi in $m^{\prime}$ 'avest $\underline{u}$ e turata. Non facilmente traducibili in canto 
appaiono inoltre le allitterazioni della sibilante che si rincorrono fino al groppo consonantico del quinto verso ( $m$ 'avestu sdegnosetta) formando una serie di paronomasie: fuss'io... chiesi ... ristoro... desiri accesi... ma se... m'avestu. Sempre sul piano della sonorità, si noterà infine che negli ultimi versi (6-9) predominano le allitterazioni e le paronomasie della " $r$ " come nei precedenti emergevano quelle della "s": una mutazione del registro espressivo che contrasta con la semplicità tonale dei testi da musicare.

Oltre al madrigale di Valerio Belli sopra ricordato (cfr. commento a 16), prossimo a questo risulta, per l'esito narrativo, il seguente dello Stigliani, presente nelle Rime del 1601 (21):

In persona d'una D. per iscusa d'alcune parole ingiuriose da lei dette al suo Amante

Non dettò il cor ciò che la lingua disse sua mentitrice ancella; innocente io son dunque, e rea sol ella.

A te ch'offeso se' punirla tocca, ma imprigionisi pria ne la tua bocca.

\section{8 - Mad. 18 - Bacio involato}

Perché fuggi tra' salci, ritrosetta ma bella, o cruda dele crude pastorella?

$5 \quad$ Miser più che felice corsi per sugger vita e morte colsi.

Quel bacio che m'ha morto, tra le rose d'Amor pungente spina, 10 La bocca involatrice, la bocca istessa che "l furò tel dice.

Nella sezione del Gareggiamento specificamente intitolata Bacio involato (già ricordata a proposito di $\mathbf{1 3}$ in cui il furto è solo desiderato), questo madrigale è riportato tronco degli ultimi due versi. Ciò indica che la riflessione del poeta sul tema, espressa dalla trasformazione del bacio-rapina in bacio-vendetta (7-9), doveva apparire completa $o$, almeno, arguta quanto basta per costituirsi come punta finale del madrigale. È possibile, comunque, che la cassatura di Carlo Fiamma (1"'Accademico Ordito" curatore della raccolta) abbia anche un'altra motivazione. Egli include, infatti, nella stessa sezione, il seguente madrigale del Guarini, proveniente dalle Rime del 1598 (con titolo Bacio rubato) da cui quasi 
sicuramente il Marino trasse ispirazione per il motivo del bacio-vendetta: la scoperta intenzione di mettere a confronto i diversi poeti scelti per l'antologia meglio si realizzava quando si potevano segnalare stretti collegamenti fra loro. Ecco il madrigale del ferrarese:

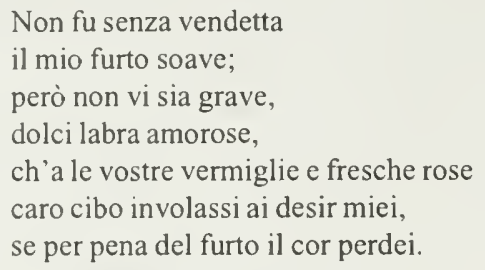

All'interno del Gareggiamento il solo impiego del tema comune da parte di diversi ovviamente non dà la certezza di debiti poetici; i quali però diventano molto probabili quando il tema è sviluppato con concetti simili $o$ addirittura identici. Nel nostro caso notiamo che Guarini apre con la vendetta e chiude con la perdita del "core", e che il Marino, stando alla riduzione del Fiamma, dopo aver narrativamente arricchito il contesto del furto con la fuga della donna (forse ricordando il bacio n. 3 di Giovanni Secondo), riprende entrambi i sottotemi, ma rovesciandone l'ordine: normalizza la perdita del "core" con la morte (senza lasciar cadere l'occasione di creare un'antitesi, 6) e dà maggior rilievo alla più rara vendetta collocandola in chiusura.

Niente a Guarini deve comunque il Nostro per quanto riguarda la struttura del madrigale. Come indica con chiarezza la distribuzione delle rime, esso è nettamente diviso in due parti (abBcdC - eFFDD), entrambe aperte da un verso con rima irrelata. La morte, che per Guarini era la punta, qui è inclusa nella enunciazione del tema, indizio questo di una costante volontà di superamento, espressa del resto, subito dopo, da una doppia chiusura epigrammatica a doppia rima baciata.

Ma c'è di più. Proprio l'autorità del Guarini, che dà esito mortale al bacio rubato, permette di credere narrativamente completo il madrigale del Nostro già all'altezza del v. 6. Con questo in mente nell'intero componimento si evidenziano un ritmo sussultorio e una narrazione paratattica marcata da tre punte in rima: bacio tolsi - morte colsi; pungente spina - mia rapina; bocca involatrice - tel dice. La scandita nettezza che queste rime evocano, dopo l'apparizione della bella pastorella in fuga tra i salci che richiama la molto celcbrata barocca fuga di Dafne, contribuì sicuramente a dare buona fortuna al madrigale in musica: dieci partiture, dal 1608 al 1641, fra cui quella del Concerto settimo (1619) di Claudio Monteverdi, ristampata quattro volte. 
19 - Mad. 19 - Bacio pubblicato con arguzia

1 Taci, bocca, deh taci

del'amate bellezze

le rapite dolcezze.

Taci, che s'egli avien che t'oda Amore,

5 la pena avrà di tue rapine il core, né minor fora ardire il parlar che "l rapire.

Ma se taciti siam quanto rapaci, avrem mill'altri e più soavi. Ah, taci.

L'arguzia di questo bacio ricevuto - un passo avanti, narrativamente, rispetto allo scherzo del bacio solo chiesto in 17 - consiste nel dire di esso, o 'pubblicario', esortando nel medesimo tempo al silenzio. Da Petrarca ai petrarchisti il poeta è spesso sdoppiato in due soggetti, sé stesso e il "core", ma qui fa coppia con la propria lingua, cui sono rivolte le argute raccomandazioni. Queste sono dettate dalla volontà di amare in segreto, ovvero senza il coinvolgimento di Amore che sempre porta con sé delle pene: un amore senza l'ingombro di Amore dunque, purificato nella sua più immediata e diretta fisica essenzialità. $\dot{E}$ in questo modo che il bacio arguto si rivela, nel distico di chiusura, giusto prologo delle nuove e più intense effusioni della Canzone dei baci.

Per quel che riguarda le fonti, sembra qui utile un rimando al seguente madrigale del Guarini, intitolato Un bacio è poco nelle Rime del '98 (n. 193) e riportato al primo posto in una partizione recante lo stesso titolo nel Gareggiamento (57r):

Un bacio solo a tante pene, cruda?
Un bacio a tanta fede?
La promessa mercede
non si paga baciando: il bacio è segno
di futuro diletto
e par che dica anch'egli, i' ti prometto
con sì soave pegno.
In tanto or godi, e taci,
che son d'Amor mute promesse i baci.

L'espressione "or godi e taci" si ritroverà identica, ma in una situazione più euforica, nel congedo della Canzone dei baci. L'invito al silenzio nell'amore, di cui Marino si ricorderà anche in un Amore secreto successivo (Ardi contento $e$ taci, CAm 23.1) è comunque ben radicato nella tradizione lirica rinascimentale. Lo si incontra in Poliziano (25), Serafino (Strambotti 409), Galeazzo di Tarsia (18), Tasso $(64,164,165,166,242,351)$, oltre che altrove nelle già ricordate 
Rime del Guarini (n. 171) e in quell'altra privilegiatissima fonte di concetti mariniani che è il canzoniere del Rinaldi (Delle Rime. Parte terza 4).

Il bacio pubblicato con arguzia piacque molto ai musicisti (diciassette partiture elencate nel Nuovo Vogel) per varie e ovvie ragioni: sintassi sciolta, lessico semplice, rime baciate facili e sonore, distribuzione dei versi a coppie alternate di endecasillabi e settenari. La binarietà nettissima dei versi e delle rime sembra contrastata dal primo settenario (abbCCddAA), ma riafferma l'ordine la vistosa ripetizione di taci. Questa voce dell'imperativo ricorre in tutti e tre i periodi che compongono il madrigale; e chiude anche l'ultimo verso, mentre rimanda al primo, facendosi portatrice di una finezza: sostituisce il sostantivo oggetto "baci" annunciato dagli aggettivi mill'altri e più soavi. Il termine quindi, in ossequio all'imposto silenzio, rimane argutamente assente in tutto il componimento. Ma solo per ripresentarsi prorompente in testa al successivo: Baci.

20 - Canz. 1 - Baci

$1 \quad$ O baci aventurosi, ristoro de' miei mali, che di nettare al cor cibo porgete; spiriti rugiadosi,

5 sensi d'amor vitali che " $n$ breve giro il viver mio chiudete; in voi le più secrete dolcezze e più profonde provo talor che con sommessi accenti,

10 interrotti lamenti, lascivetti desiri, languidetti sospiri tra rubino e rubino Amor confonde e più d'un'alma in una bocca asconde.

15 Una bocca omicida dolce d'amor guerrera, cui natura di gemme arma ed inostra, dolcemente mi sfida e schiva e lusinghiera

20 ed amante e nemica a me si mostra. Entran scherzando in giostra le lingue innamorate; baci le trombe son, baci l'offese, baci son le contese;

25 quelle labbra ch'io stringo 
son l'agone e l'arringo;

vezzi son l'onte e son le piaghe amate, quanto profonde più, tanto più grate.

Tranquilla guerra e cara

30 ove l'ira è dolcezza, amor lo sdegno e ne le risse è pace; ove 'l morir s'impara, l'esser prigion s'apprezza, né men che la vittoria il perder piace.

35 Quel corallo mordace che m'offende mi giova; quel dente che mi fere adora adora, quel mi risana ancora;

40 di vita mi raviva; ond'io, c'ho nel morir vita ognor nova, per ferito esser più, ferisco a prova.

Or tepid'aura e leve, or accento, or sorriso

45 pon freno al bacio a pien non anco impresso.

Spesso un sol bacio beve sospir, parola e riso; spesso il bacio vien doppio, e "l bacio spesso tronco è dal bacio stesso.

$50 \quad$ Né sazio avien che lasce pur d'aver sete il desir troppo ingordo: suggo, mordo, rimordo, un bacio fugge, un riede, un ne more, un succede;

55 dela morte di quel questo si pasce e pria che mora l'un, l'altro rinasce.

L'asciutto è caro al core, il molle è più soave, men dolce è quel che mormorando fugge.

60 Ma quel che stampa Amore d'ambrosia umido e grave i vaghi spirti dolcemente sugge.

Lasso, ma chi mi strugge ritrosa il mi contende

65 in atto sì gentil che 'nvita e nega, ricusa insieme e prega.

Pur amata ed amante e baciata e baciante alfin col bacio il cor mi porge e prende 
70 e la vita col cor mi fura e rende.

Miro, rimiro ed ardo,

bacio, ribacio e godo

e mirando e baciando mi disfaccio.

Amor tra "l bacio e "l guardo

75 scherza e vaneggia in modo

ch'ebro di tanta gloria i' tremo e taccio;

ond'ella che $m$ 'ha in braccio,

lascivamente onesta,

gli occhi mi bacia e fra le perle elette

80 frange due parolette:

"cor mio" dicendo; e poi

baciando i baci suoi,

di bacio in bacio a quel piacer mi desta

che l'alma insieme allaccia e i corpi innesta.

85 Vinta allor dal diletto

con un sospir sen viene

l'anima al varco e il proprio albergo oblia;

ma con pietoso affetto

la 'ncontra ivi e ritiene

90 l'anima amica che s'oppon tra via;

e 'n lei, ch'arde e desia

già languida e smarrita,

d'un vasel di rubin tal pioggia versa

di gioia, che sommersa

95 in quel piacer gentile

cui presso ogni altro è vile,

baciando l'altra ch'a baciar la 'nvita,

alfin ne more e quel morir è vita.

Deh taci, o lingua sciocca,

100 senti la dolce bocca

che ti rappella e dice: - Or godi e taci -

e per farti tacer raddoppia i baci.

Questa canzone, cui giusta secolare fama ha assegnato un primato assoluto di rappresentatività del barocco letterario, e vuoi pure, talvolta, di segno negativo, è innanzi tutto da riconoscere come vera sintesi dell'esperienza poetica osculatoria del Marino.

Sulla sua data di composizione, più che le indicazioni fornite dal poeta stesso nella lettera dedicatoria a Tomaso Melchiorri ("scherzo giovanile, e poco men che fanciullesco") dicono i vistosi debiti nei confronti della "canzonetta" Nettar dolce trabocca del Rinaldi, uscita a stampa nel 1590. Certo è, inoltre, che la canzone era già nota ben prima che il Marino lasciasse Napoli nel 1600, come 
afferma il Borzelli (10) ricorrendo alla testimonianza del Loredano. E poteva il poeta anche vantare, sempre al Melchiorri nel licenziare le prime rime, "ch'ella è stata da molti nobili intelletti trasportata in vari linguaggi, come Schiavone e Spagnuolo, e pur hora da Mons. Ruberto Crampone leggiadrissimamente in Francese". Alla inusitata fortuna letteraria del componimento non ancora a stampa, fa riscontro l'immediato favore dei musicisti dopo la pubblicazione: Il Nuovo Vogel registra otto partiture, di cui tre (di Antonio il Verso, Benedetto Magni e Salomone Rossi) dell'anno 1603. Manca l'indicazione di quella di Tommaso Pecci, l'unico compositore ricordato dal Marino, che evidentemente dovette precedere la stampa delle Rime se a lui dedicato troviamo un sonetto di ringraziamento incluso nelle Varie (A Va 12), per aver "messo in canto" il componimento.

Baci è la prima "canzone" tra quelle annunciate nel frontespizio ("Madrigaili e Canzoni"). Che il termine sia da intendere con una certa elasticità ce lo dice la presenza, alla fine della raccolta, dei due componimenti in ottava rima inclusi tra le "canzoni". Ma c'è da fare un'altra distinzione: tra le canzoni vere e proprie, di chiara tessitura petrarchesca, come questa e 31 per restare nella sezione dei baci, e le canzonette o 'odicine', come 27 e 28 , più prossime a quelle del Casoni che a quelle del Chiabrera, composte da stanze brevi e rime facili. Mancano studi specifici sui rapporti tra le due forme in Marino; qui ci si limita a rilevare che esse continuano a coesistere nella Lira del ' $14 \mathrm{e}$ che in entrambe è notevole l'insistenza sulle rime baciate (praticamente di regola nel distico conclusivo, che precede di solito rime alterne nelle canzonette e altre rime baciate nella sirima delle canzoni).

Come s'è detto, il componimento rappresenta, con il suo amplesso finale, l'approdo narrativo del primo dei due segmenti che compongono la sezione dei baci. Ma c'è anche una narrazione interna al componimento stesso, e su di essa conviene subito puntare l'attenzione, sia per registrare la struttura organizzativa della canzone, e sia per introdurre il confronto con il ben identificabile doppio ipotesto costituito dalle tematicamente parallele canzoni del Guarini e del Rinaldi.

La prima stanza funge da introduzione. La generica esclamazione iterata nella fronte ( $O$ baci avventurosi ... spiriti rugiadosi) è seguita nella sirima da un diretto riferimento a sensazioni personali del poeta (le piì secrete / dolcezze... / provo talor) che indicano un alto livello di coinvolgimento nella 'storia', ovvero nell'esperienza osculatoria che egli si accinge a narrare. Il racconto dei 'fatti' ha inizio nella seconda stanza, con i primi gesti e la definizione del setting (Una bocca omicida / ... / dolcemente mi sfida / entran scherzando in giostra / le lingue innamorate). Nella terza stanza il gioco erotico si intensifica con figurazioni guerresche dipendenti dalla precedente metafora del duello, che conducono prima al labbro che offende e giova e al dente che fere e risana, e quindi al bacio tutt'intero che priva di vita e 
ravviva. Una breve interruzione dell'attività osculatoria, dovuta al respiro, a qualche parola e ai sorrisi (or tepid'aura e leve / or accento or sorriso / pon freno al bacio), si registra all'inizio della quarta stanza. Ma il gioco riprende subito e con maggiore intensità fino alla fine della stanza successiva quando si incontra lo scambio euforico dei cuori che è indizio certo dell'approssimarsi dell'amplesso vero e proprio, se è vero che "core", come facili e numerosi esempi precedenti e successivi attestano, è metafora sessuale (Alfin col bacio il cor mi porge e prende / e la vita col cor mi fura e rende). Si arriva cosi, nella sesta stanza, tra baci voyeristici e fremiti d'ebrezza, a quell'innescatissima esclamazione della donna ("cor mio") che fa da immediato preludio all'"innesto" dei corpi. Nella settima e ultima stanza si passa dalla copula all'orgasmo e alla eiaculazione (Vinta allor dal diletto / ... sen viene / l'anima / ...e 'n lei [nell'"anima amica"]... / d'un vasel di rubin ... pioggia versa / di gioia ... sommersa / in quel piacer gentile / cui presso ogni altro è vile).

Come di altri casi esemplari di poetica mariniana, e viene a mente la riscrittura intarsiata della storia di Psiche nel quarto canto dell'Adone, anche di questa canzone si possono indicare precise e copiose fonti di ispirazione. Inappropriato sarebbe qui portare in campo il termine 'furto' pur nella meno della nostra inibita accezione barocca, perché i componimenti del Guarini e del Rinaldi, cui tanto deve questa canzone, vuoi per la notorietà degli autori e vuoi anche per $\mathrm{i}$ loro indiscutibili meriti intrinsici, non costituivano certo materiale da rapina. Se su di essi si esercita il famigerato rampino è con diversa motivazione. Sarà da parlare allora, si propone, di decisa volontà di superamento da parte del Nostro, pur con l'intenzione di rimanere solidamente ancorato agli schemi della tradizione lirica più fruiti nel suo tempo: un'operazione, questa, di cui ha parlato in tempi recenti Alessandro Martini (cfr. "Marino postpetrarchista"). Ecco le bellissime fonti principali della canzone dei baci; si riportano per intero per facilitare il raffronto, visto anche che mancano del tutto edizioni moderne.

\section{Battista Guarini}

$1 \quad$ Baci soavi e cari

cibi della mia vita

ch'or m'involate or mi rendete il core, per voi convien ch'io impari

5 com'un'alma rapita non sente il duol di morte e pur si more.

Quanto ha di dolce Amore perch'io sempre vi baci, o dolcissime rose,

10 in voi tutto ripose; e s'io potessi ai vostri dolci baci la mia vita finire, 
o che dolce morire.

Baci amorosi e belli,

15 mentre che voi m'aprite

di rubini e di perle alti tesori,

e tra questi e tra quelli

aure dolci e gradite

spirano di vitali arabi odori,

20 l'alme dei nostri cori

parton da la radice

e su le labia estreme

l'una e l'altra si preme,

e bascia e stringe e sospirando dice:

25 Amor ch'unisce l'alme

unirà anche le salme.

Baci affamati e ingordi,

ai cui misti diletti

né mai si satia Amor né mai respira,

30 tu dente avido mordi

e tu lingua saetti

e mormorando parli, il cor respira.

Intanto il guardo mira,

e mentre ognun pur vuole

35 mordere e sospirare

e vedere e baciare,

baci morsi sospir guardi e parole

fan sì dolce concento

che vi sta il cielo intento.

40 Baci cortesi e cari,

e voi labri amorosi,

che tanto date altrui quanto togliete,

chi v'ha così infiammati

de' miei? Chi si bramosi

45 vi fa di quello onde sì ricchi siete?

Rose d'amor ch'avete

d'ogni bellezza il vanto,

ben riconosco il dono

per voi sì dolce sono:

50 baciate questi pur che da voi quanto

in me si cura e prezza

tutto è vostra dolcezza.

Baci oimé non mirate

che mentr'io parlo oblio

55 come l'ora sen va fugace e breve. 
Baciate oimé baciate, lungo è il nostro desio, ma la speranza è frale e 'I tempo breve. Taccia chi gioir deve,

60 baci non siate lenti, venite a mille a mille quante son le faville del mio bel foco, e quanti raggi ardenti mia luce han gli occhi vostri

65 sian tanti i baci nostri.

Baci, di tante gioie una sol resta che tutte l'altre avanza sola del cor speranza. ${ }^{3}$

\section{Cesare Rinaldi}

\section{Canzonetta}

1 Nettar dolce trabocca

dai rubini amorosi, mentre la lingua scocca come da arco stral baci focosi.

5 Amor infonde e mesce due lingue in una lingua, e ' $n$ mezzo ai baci cresce il desir di baciar non che s'estingua; stansi le labbra unite,

10 come il desio lor chere, e fra perle gradite corron le lingue a farsi prigioniere.

Nulla amarezza è mista fra i dolcissimi baci,

15 nulla il pensiero attrista, e più crescon d'Amor l'eterne faci; volan ardenti insieme l'alme a baciarsi anch'elle, e fra dolcezze estreme

20 sembran sui vivi fiori api novelle.

Ogn'aspro cor si molce, ogn'alma s'innamora, e "I bacio è via più dolce che di colombe all'apparir di Flora. 
talor lievi aure sono, stansi le labbra a segno qual veloce destrier ch' attenda il suono; quivi è desio di foco,

30 d'Amor son le faville, tornano al primo gioco, e raddoppiansi i baci a mille a mille; suonan fra i colpi impressi voci d'amor ripiene

35 e " $n$ un fan di se stessi i rubini ai rubin mille catene.

More il bacio e sen fugge, nasce il bacio e sen riede, entra il primo e si strugge,

40 caro figlio il secondo è fatto erede; fassi il gioire alterno vita ai sensi vitale, e fra le gioie eterno sui baci Amor va ventilando l'ale;

45 corre il bacio e s'innesta di bei coralli a paro, indi s'interna e resta quanto profondo più tanto più caro.

11 molle è dolce al core,

50 insipido è l'asciutto, in bel giardin d'Amore son fiori i baci in cui germoglia il frutto; già verdeggiante pende, felice chi lo coglie,

55 già il piè la cima ascende, e lo sterpe la mano, il sen l'accoglie; vien maggior il diletto, segue il languir soave, manca ai baci l'oggetto

60 e la sete al desir cresce più grave.

Canzone il bacio è fiamma che incende e non consuma, se in esso altri s'infiamma ebbro sen vola al ciel qual lieve piuma.

(Delle Rime. Parte terza 35-38)

Non è qui il caso, per brevità, di indicare la misura precisa dei debiti mariniani che, del resto, il lettore di buona volontà può calcolare da sé; pare utile però insistere sulla sopra menzionata volontà di superamento in Marino. Si consideri 
innanzi tutto il numero delle stanze, sette in Marino e cinque in entrambi gli altri; e poi il numero di versi che le compongono, dodici in Rinaldi, tredici in Guarini, quattordici nel Nostro. Tutte e tre le canzoni hanno un commiato, ma quello di Marino è l'unico in cui si fa specifico riferimento a un evento narrativo (e cioè all'appagamento dei sensi verificatosi nell'ultima stanza), che ci pare la chiave necessaria per una corretta interpretazione della canzone. Anche Rinaldi giunge, con la metafora del frutto che germoglia dal bacio (e lo sterpe la man, il sen l'accoglie; / vien maggior il diletto / segue il languir soave), all'unione sessuale; ma la sua "canzonetta" ha un carattere decisamente più descrittivo che narrativo, come è provato dal fatto che il poeta non partecipa in prima persona all'“evento" esposto (si legge, per esempio, nell'ultima stanza: ... il frutto / già verdeggiante pende,/ felice chi lo coglie, dove il "chi" è un generico e impersonale fortunato amante. E, ancora, distaccato è il tono definitorio del commiato: Canzone, il bacio è fiamma / che incende...). Per quel che riguarda Guarini, è solo nel commiato che si incontra un vago riferimento al rapporto sessuale (baci, di tante gioie una sol resta / che tutte l'altre avanza / sola del cor speranza). Ma nel ferrarese il Marino ha raccolto, oltre a vari concetti e a locuzioni espressive (come in Rinaldi, che in questo è stato comunque saccheggiato in maniera più sistematica), la tessitura stessa della canzone (identiche sono la fronte e la prima parte della sirima), l'incipit esclamativo (che Guarini mantiene in ogni stanza), e il tono di coinvolgimento personale (Guarini: O baci soavi e cari / cibi della mia vita. Marino: $O$ baci avventurosi / ristoro dei miei mali). E si noti infine, per restare sempre sugli elementi di confronto più vistosi, come il cibo del Guarini ritorni nel terzo verso mariniano, che di nettare al cor cibo porgete, insieme al nettare che apre la canzone del Rinaldi, Nettar dolce trabocca.

Siamo di fronte a un componimento che nella ripetizione delle forme, nella selezione dei concetti e nell'intarsio semantico rinnova e consolida i modi e i contenuti della tradizione lirica, mentre un superamento effettivo della poetica comune si attua sul fronte della narrazione episodica. Il Marino attinge idee, espressioni, sintagmi, come materiali da costruzione e, senza sentire alcun obbligo di aderenza ai contesti o anche ai significati originali della citazione, marcia sicuro nella direzione che si è scelto per l'impianto narrativo, badando, soprattutto, alla leggerezza del dettato. E così, per esempio, raccoglie questo distico dal Rinaldi, il molle è dolce al core / insipido è l'asciutto, e lo 'corregge' eliminando il termine insipido (presente anche altrove nel bolognese, Insipida mi baci, in Delle Rime. Parte terza 22) evidentemente troppo duro per i suoi baci: L'asciutto è caro al core / il molle è più soave.

Per dare una sommaria idea del macrotesto di cui questa canzone è parte emersa come di un gigantesco iceberg, si indicano di seguito, sinteticamente, alcuni altri riscontri tematici e lessicali. 
vv. 5-6 Sensi d'Amor vitali / che 'n breve giro il viver mio chiudete

Ai sensi vitali che compaiono nella "canzonetta" del Rinaldi, segue il breve giro della vita presente nel Pastor fido: E la mia vita, chiusa / in cosi breve spazio, I non era che un bacio (2.1.197-99).

\section{v. 10 interrotti lamenti}

A questi lamenti erotici corrispondono, in un contesto simile, le voci interrotte del Rinaldi (Delle Rime. Parte terza 169).

vv. 13-14 Tra rubino e rubino Amor confonde / e più d'un'alma in una bocca asconde

Le anime che si confondono nel bacio sono un tema neoplatonico esplicitato da Pico della Mirandola e successivamente raccolto dal Firenzuola prima di diventare stereotipo petrarchista (cfr. Perella, 169-213). Giovanni Secondo riporta il concetto nel bacio n. 10. In area più prossima al Nostro si trovano affinità in Casoni (Ode 31), Rinaldi (Delle Rime. Parte terza 139; Gareggiamento 62r).

vv. 15-28 Una bocca omicida / ... mi sfida ... / quelle labbra ch'io stringo / son l'agone e l'arringo

La giostra osculatoria compare in Tasso (Rime 183, 214), Casoni (Ode 31), Rinaldi (Delle Rime. Parte terza 70, 120; Gareggiamento 61v). La bocca omicida richiama le omicide labbra del Guarini (Pastor fido 2.1.233).

vv. 35-42 quel bacio che mi priva / di vita mi raviva

Tema legato al già visto bacio in cui si confondono le anime degli amanti. Un altro riscontro è in Rinaldi: Già per due labra fui / di vita e d'alma privo, / hor fan due labra ch 'io ritorno vivo (Gareggiamento $58 \mathrm{v}$ ).

vv. 53-56 Un bacio fugge, un riede, / un ne more, un succede; /... / e pria che more l'un, l'altro rinasce

Oltre a un distico della "canzonetta" riportata (vv. 37-38: More il bacio, e sen fugge, / nasce il bacio, e sen riede), Rinaldi offre quest'altro verso al Marino: e, mentre un bacio mor, l'altro rinasce (Delle Rime. Parte terza 80).

vv. 69-70 Alfin col bacio il cor mi porge e prende / e la vita col cor mi fura e rende

La diffusione capillare del tópos è confermata dal fatto che Casoni può omettere il sostantivo oggetto in questi versi: Baci ch'altrui togliete / quel che dolce 
baciando anco rendete (Ode 59). Ma Marino, come si suggeriva sopra, dà corpo sessuale al vago "core" affidandogli cosi un' ardita valenza metaforica.

vv. 71-72 Miro, rimiro ed ardo, / bacio, ribacio e godo

11 distico, che sarà ricordato nell' Adone (Bacia e dopo il baciar mira e rimira / le baciate bellezze or questi or quella [8.124]), trova riscontri in Rinaldi: miro e mirando godo (Delle Rime. Parte terza 53); Mentre bacio e ribacio (Gareggiamento 62r).

v. 81 "cor mio"

L'esatta espressione riapparirà in circostanze analoghe nell'Adone (8.146).

v. 84 che l'alme insieme allaccia e i corpi innesta

Le anime si allacciano in Casoni (Ode 31) e i corpi si innestano in Rinaldi (Delle Rime. Parte terza 141).

vv. 97-98 [L'anima] baciando l'altra ch'a baciar la 'nvita / alfin ne more, e quel morire è vita

Si trovano riscontri in Giovanni Secondo (Basium n. 13, già riportato nel commento a 13), e in Tasso: Amorosa fenice / nel sol che solo adoro / ardendo vivo e moro, / e morendo rinasco... (Rime 290).

vv. 99-102 Or godi e taci

Pare ovvio un ricordo del Guarini, intanto or godi e taci (Rime, n.193), dal madrigale già citato per esteso a proposito di 19. Ma l'esortazione è piuttosto comune; si veda, per esempio, anche Rinaldi (Delle Rime. Parte terza 70, 107).

21 - Mad. 21 - Baci cari

1 Tornate, o cari baci, a ritornarmi in vita, baci al mio cor digiuno esca gradita.

Voi, di quel dolce amaro

5 per cui languir m'è caro, di quel vostro non meno nettare che veneno, pascete i miei famelici desiri, baci, in cui dolci provo anco i sospiri. 
L'incipit di questo madrigale, immediatamente a ridosso dei più famosi baci, vistosamente richiama l'esperienza appena vissuta. Che di una coda si tratti, è confermato da alcune ricorrenze: torna, ma in modo più pacato e distante, il concetto del bacio che ridà la vita, prima ampiamente sfruttato; e torna anche la metafora del bacio-cibo posta qui in apertura (con riferimento al cor già digiuno) come nella canzone. Il nutrimento era li nettare, raccolto nella "canzonetta" del Rinaldi, e qui anche il nettare riappare (di quel vostro non meno / nettare che veneno), ma probabilmente mutuato da quest'altro passo dello stesso poeta bolognese: entra l'umida lingua e seco a paro / fora il nettar veleno, assenzio il mele (Delle Rime. Parte terza 200). Canzone sorella dei Baci, infine, è quella estravagante dei Sospiri (cfr. Simon; Taddeo) e l'associazione dei due temi risulta qui ben espressa visto il rilievo assegnato a quest'ultimo con la collocazione nella punta finale.

Tipico madrigale cantabile - il Nuovo Vogel ne riporta ben diciassette versioni musicali - il componimento risulta metricamente composto da quattro coppie di versi a rima baciata, precedute da un verso con rima irrelata, in cui però si evidenzia il termine chiave baci che è nel titolo, e che ricompare sulla pagina una terza volta: nell'ultimo verso, ben isolato, a una corretta lettura, fra due forti pause. Il madrigale è sintatticamente ordinato in due periodi diversi, secondo lo schema mariniano più comune; le novità narrative rispetto ai baci prececenti sono espresse soprattutto nella seconda parte in cui troviamo le prime avvisaglie (amaro, languir, veneno, sospiri) di quella ansietà post coitum che esploderà negli Amori notturni.

22 - Mad. 21 - Baciator dubbioso

$1 \quad$ Vorrei baciarti, o Filli, ma non so prima ove il mio bacio scocchi, nela bocca o negli occhi.

Cedan le labra a voi lumi divini,

5 fidi specchi del core, vive stelle d'Amore.

Ahi, pur mi volgo a voi perle e rubini, tesoro di bellezza, fontana di dolcezza,

10 bocca, onor del bel viso: nasce il pianto da lor, tu m'apri il riso.

Interrogandoci sulla calcolata dispositio dell'intera sezione dei baci, abbiamo già assegnato a questo la posizione mediana; aggiungiamo ora che si tratta di un centro vuoto, per cosi dire, a causa della caduta di tensione tra il desiderio del bacio e la sua realizzazione. La sequenza narrativa precedente era stata 
motivata dal desiderio irrefrenabile del poeta che aveva condotto prima al furto e poi allo scambio voluttuoso dei baci. La narrazione è ora interrotta da un dubbio che lascia perplesso il baciatore; ma l'impasse sarà presto sciolta, dall'ormai attivato erotismo della donna, nel madrigale seguente dove, appunto, la 'storia' ricomincia. Se in termini di plausibilità narrativa non si può fare a meno di notare l'improbabilità della motivazione della sosta, tutt'altro che peregrine si devono giudicare in termini teorici le ragioni del dubbio, cui il poeta ritornerà, nel pieno dell'azione, in 26. Si tratta di stabilire tra vista e tatto quale sia il senso più importante. Pur senza rinunciare alle delizie del primo, Marino opta per il secondo. La scelta è confermata nell'Adone (cfr. 8.19-20, ma si ricordi anche l'intera struttura del palazzo di Amore) dove al tatto sarà chiaramente assegnata la preminenza non solo sulla vista, ma su tutti gli altri sensi, per "polemica nei confronti... [della] classifica neoplatonica" (Pozzi 397).

Il madrigale consta di tre periodi: i primi due di tre versi ciascuno, il terzo di cinque. Nel primo viene posto il dubbio; nel secondo si espongono le ragioni della vista; nel terzo quelle prima di pari merito ( 8 e 9 come 5 e 6 ) e poi vittoriose (10-11) del tatto. La binarietà ricorrente, nonostante l'insistenza delle rime baciate $(\mathrm{Bb}, \mathrm{dd}, \mathrm{fF})$, è più semantica che strutturale per l'alternarsi dei termini riferiti a occhi e bocca.

Per quanto riguarda le fonti, vanno innanzi tutto citati questi versi conclusivi di un bacio del Secondo: Heu, quae sunt oculis meis / Nata proelia cum labris? / Ergo ego mihi vel lovem / Rivalem potero pati? / Rivales oculi mei / non ferunt mea labra (Basia, n. 7). Si ricordano poi, per l'affinità del tema, due componimenti del Tansillo, la canzone Canta i divini occhi splendenti e la bella bocca armoniosa della sua donna (Canzoniere, canz. 1), e il sonetto Non sa celebrar degnamente gli occhi splendenti e sereni e la bocca celeste della sua donna (son. 37). Gli specchi del core sono due volte nelle Rime del Tasso (88, 221). Il distico conclusivo, bocca... / ... tu m'apri il riso, richiama la vittoria / d'Amor nasce dal riso del Casoni (Ode 29). Schede interessanti si raccolgono in una brevissima sezione del Gareggiamento (due madrigali, di Alessandro Ragarin e Carlo Fiamma) intestata a Labra dolci, luci amare: il titolo dice tutto. E si menziona infine il Rinaldi che sorprendentemente coglie insieme tatto e udito (Delle Rime. Parte terza 32) e udito e vista (Delle Rime. Parte terza 79).

Rime facili e baciate, sintassi piana e collaudate cantabilissime metafore contribuirono alla fortuna di questo madrigale in musica: il Nuovo Vogel ne indica quindici partiture.

23 - Mad. 22 - Bacio mordace

$1 \quad$ Eccomi pronta a i baci:

baciami, Ergasto mio, ma bacia in guisa 
che de' denti mordaci

nota non resti nel mio volto incisa,

perch'altri non $\mathrm{m}$ 'additi e in esse poi

legga le mie vergogne e i baci tuoi.

Ahi, tu mordi e non baci,

tu mi segnasti, ahi ahi;

possa io morir se più ti bacio mai.

La seconda fase della vicenda osculatoria riprende con questo morso di passione dato in risposta alle sollecitazioni della donna. I baci mordaci sono per il poeta legati sempre a situazioni di prorompente sessualità, come attestano gli esempi in A Bo 86 e nell' Adone $(7.85 ; 7.147$; 7.244; 8.125-26; 15.14; 18.238). Sul fronte della narrazione, infatti, notiamo il riattivarsi della tensione erotica che, dopo aver condotto all'amplesso di $\mathbf{2 0}$, era apparsa contenuta, per motivi diversi, in 21 e 22 . Il ritmo sussultorio di questa fase del racconto, intonato all'alternarsi dei baci come fossero tanti colpi di scherma, secondo la metafora sfruttatissima in 20, si estende ai componimenti successivi: dopo la cortese pausa delle scuse (24), si rialza la tensione con la Tempesta di dolcezza di 25, in cui troviamo, tra l'altro, un "bacio mordace" della donna (7-8) che vanifica il proposito di castità della chiusa del componi-mento che stiamo considerando.

Come perfettamente espresso nella mirabile versione musicale del Monteverdi, il madrigale è nettamente scandito in due parti (1-6; 7-9) segnate, all'inizio, da un'esclamazione; la quale è peraltro, in entrambi i casi, contenuta in un verso chiuso dall'identico termine, baci, in rima equivoca.

Nel nome del baciatore si può leggere un omaggio al Sannazaro o al Guarini, oppure a entrambi, ricordando comunque quei Sospiri d'Ergasto, confluiti nella Sampogna, cui sappiamo il Marino aveva già lavorato nel periodo napoletano.

I riscontri testuali devono cominciare dal Secondo, che offre due baci mordaci (Basia, nn. 5, 8). Del Guarini, oltre ai morsi della sua già vista canzone dei baci, parallela a quella del Marino, si ricorda un passo del dialogo tra Ergasto e Mirtillo in cui quest'ultimo dice: Io, poi ch 'a morte mi sentii ferito, / come suol disperato, / poco mancò che l'omicide labbra / non mordessi e segnassi (Il pastor fido 2.1.231-34). Del Casoni sorprende il verso Eccomi pronto ai baci (Ode 31). Schede utili, come al solito, si trovano nel Gareggiamento che dà i Baci morsicati di Giovan Battista Leoni, Gasparo Murtola e Gioan Maria Guizzardi (65r-65v), oltre a questi versi del Petracci in un'altra sezione (Baci dolci): ai ai, tu m'hai ferito / Filli, tu m'hai tradito (56v).

Notevole l'interesse dei musicisti - 15 partiture nel Nuovo Vogel motivato sicuramente, oltre che dalle rime facili e, con esclusione di -oi (5-6), tutte recanti le stesse vocali, dall'alto livello di espressività del madrigale. 
24 - Mad. 23 - Scusa di bacio mordace

1
Al desir troppo ingordo
perdona, o Cinzia; e s'io ti suggo e mordo, scusa la fame ardente
ch'alletta al cibo suo l'avido dente.
ch'io macchi il volto tuo co' baci miei;
ché l'altra Cinzia ancor, la dea di Delo,
ha pur tinto di macchie il volto in cielo.

5 Né tu lagnar ti dei

Cosi come al bacio chiesto (16) faceva seguito uno scherzo di bacio chiesto (17), e al bacio involato (18) un'arguzia sulla perpetrata rapina (19), qui troviamo apposta una scusa al bacio mordace appena dato. Queste coppie marcano momenti di via via più intensa intimità nella vicenda osculatoria e presentano una doppia valenza strutturale: da una parte sono un'altra indicazione del costante ricorso alla binarietà, e dall'altra confermano l'inarrestabile dinamismo della narrazione.

Il madrigale consta di quattro distici a rima baciata; ognuno dei primi tre è costituito da settenario ed endecasillabo, mentre il quarto, che porta la punta, è espresso da una coppia di endecasillabi. Dà varietà al ritmo, oltre a ciò, la forte cesura del secondo verso (endecasillabo a minore) che fa del secondo emistichio un settenario a sé stante.

Il nome della donna è particolarmente significativo. Cinzia è, innanzi tutto, nome diverso da Filli, incontrato in 22. Notiamo poi, nei componimenti successivi, altre variazioni: Clori in $\mathbf{2 7}$, di nuovo Filli in 28 e infine, in 31 , Lilla. Compagna poetica più costante del Nostro, quest'ultima non solo compare più volte nell'intera Lira (con presenze numerose nelle Marittime e negli Amori), ma si ritrova anche nella Sampogna, in cui è La bruna pastorella (primo dei secondi idilli) e nell' Adone, in cui si ufficializza la sua posizione a fianco a Fileno/Marino (cfr. 9.48; 9.54; 20.71-72; 20.75). I diversi nomi della donna attestano la genericità di riferimento dei baci poetici e mettono quindi in guardia, se ce ne fosse bisogno, contro una semplicistica loro interpretazione come mero resoconto di una esperienza di vita. La Cinzia, o Diana, o Luna, che qui appare può anche intendersi pronuba dei prossimi pastorali amori $(\mathbf{2 7}, \mathbf{2 8})$; è comunque nuda di fronte al pastore Endimione in 31 (26-28), quando nella canzone l'enfasi cadrà sulle immagini notturne che accompagnano il turbamento amoroso. Ma ovviamente Cinzia in questo madrigale è soprattutto chiamata in causa per l'arguta somiglianza delle macchie lunari con i segni lasciati sul suo volto dal bacio mordace. Si coglie l'occasione qui per ricordare l'interesse mariniano per l'astronomia, attestato sia dalla lode del Galilei nell'Adone (10.39-47) che include una descrizione della superficie lunare, sia dalla corrispondenza con un amico dello scienziato, l'ottico veneziano 
Girolamo Magagnati, di cui danno certa notizia le Lettere di quest'ultimo raccolte in un manoscritto della Thomas Fisher Rare Book Library di Toronto. ${ }^{4}$

Ai già citati riferimenti al tema del bacio mordace si possono qui aggiungere alcuni minimi riscontri testuali. Il sintagma desiri ingordi ricompare nell' Adone (5.46), mentre $\mathrm{i}$ due termini che lo compongono appaiono in significativa prossimità nelle Rime del Bandello (ingorda e desiosa vista, 157.34), in quelle di Vittoria Colonna (bramose ingorde voglie. / ... bei nostri desiri, 97.8-9) e nell'Adone ancora $(7.5 ; 14.12)$. La fame ardente è in Celio Magno (A questa tua, Signor, sacrata mensa /... / vengo per ristorar mia fame ardente, 133.3). L'avido dente è nella già vista canzone dei baci del Guarini ( $t u$ dente avido mordi, 30). Le macchie lunari rimandano alle Rime del Tasso $(1098,1555)$ e all'Adone in cui ... la luna è macchiata e il sol s'eclissa.

Il Nuovo Vogel riporta solo tre versioni musicali di questo madrigale. Il contenuto entusiasmo dei musicisti è probabilmente da imputare all'abbassamento di vivacità espressiva che si registra nella seconda parte.

\section{5 - Mad. 24 - Baci dolci}

$1 \quad$ Tempesta di dolcezza

su l'anima mi versa

Amor mentr'io ti bacio, o mio tesoro.

Lasso lasso ch' io moro!

5

Un diluvio di baci l'ha sommersa:

già di quel labro al tuon dolce sonoro

dietro al lampo d'un riso

m'ha del tuo dente la saetta ucciso.

Tonifica la "mordacità" dichiarata nei due madrigali precedenti la "dolcezza" di questo; che è comunque "tempestosa", a conferma di un aumento progressivo della tensione erotica (regolarmente espressa da termini antitetici), del resto rilevabile nella "saetta del dente" della donna (8).

Il componimento, di otto versi, è diviso in due parti di quattro ciascuna: la prima, tre settenari e un endecasillabo, va da tempesta a moro; la seconda, tre endecasillabi e un settenario, da diluvio a ucciso. Nella prima bacia l'uomo; nella seconda, mordacemente, la donna. Entrambe le parti presentano in coda rime baciate, portate da termini che mostrano una certa opposizione semantica: tesoro / moro; riso / ucciso.

Dominano le metafore atmosferiche: tempesta (1), diluvio (5), tuon (6), lampo (6). L'arguzia è però soprattutto affidata al tuono perché esso vale anche come "tono musicale" o, semplicemente, "parole". In quest'ultimo senso aveva adottato il tuono Serafino Aquilano in questo notevole sonetto: 
Se ben resposi a tue parol faconde, avendo a ogni altro tuon l'orecchie sorde, è che due alme in noi son sì concorde che la tua voce in me loquela infonde.

L'exempio in doi strumenti non se asconde, che s'egli advien che l'un con l'altro accorde quella union delle sonore corde fa che toccando l'un l'altro responde.

Da che tua fama sì lontan mi punge teco fu el mio valor sì forte unito, come fiamma con fiamma si congiunge;

tal che quel tuon de la tua bocca uscito un'ecco forma in me da presso e lunge e ciò ch'io parlo ho da tua bocca odito. (Rime. Son. 23)

Non risultano riscontri testuali significativi delle metafore atmosferiche così combinate, mentre il lampo d'un riso è forse un ricordo del Tasso (e quel rossore e 'l riso / ne l'angelico viso / d'in bel lampo credea purpurea luce [Rime 263]); e ricomparirà nell'Adone (e d'onesto sorriso un lampo dolce [11.76]).

Le undici partiture segnalate dal Nuovo Vogel attestano una buona fortuna del madrigale fra i musicisti; che pare giusto ascrivere, oltre che alle rime facili e baciate, alla novità delle immagini.

26 - Mad. 25 - Sguardi e baci

$1 \quad$ Qualor, labra soavi, e vi miro e vi bacio, l'un l'altro senso invidia, ond'a tutt'ore questo e quel si confonde,

5 e spesso il bacio a guardo, il guardo al bacio le dolcezze profonde, qual geloso rival, fura ed asconde.

Se miro, allor bram'io baciar; se bacio, allor mirar desio.

10 Potesser per miracolo d'Amore o il guardo o il bacio scocchi e mirarvi la bocca e baciar gli occhi. 
La tematizzazione della sinestesia, con vista e tatto che si invidiano a vicenda (3) e si confondono (4), richiama espressamente le riflessioni del Baciator dubbioso (22). Ma qui, come già si è avuto modo di notare, non c'è una pausa narrativa che interrompe lo sviluppo dell'azione: siamo nel mezzo delle nuove effusioni amorose, repentinamente riattivate dal Bacio mordace (23), che condurranno ai ripetuti amplessi degli Amori notturni (31). Conferma questo andamento generale del racconto una minima, ma sicura spia dell'incremento di tensione erotica: quella dolcezza che sgorga dalla bocca della donna come acqua da fontana in 22 (9), che diventa una tempesta al bacio in 25 (1-3), e che troviamo qui ben consolidata e al plurale (profonde dolcezze, 5-7) per il coinvolgimento attivo e simultaneo dei due sensi in questione.

A differenza di 22 , in cui dopo l'introduzione (1-3) i due sensi venivano considerati separatamente, qui abbiamo una voluta e persistente mescolanza dei termini che a essi si riferiscono; il che giustifica, strutturalmente, la dispositio di tre diversi segmenti, di progressiva complessità, in ognuno dei quali rileviamo la compresenza di iterazione e chiasmo: il bacio al guardo, il guardo al bacio (6); se miro, allor bram'io / baciar; se bacio, allor mirar desio (8-9); o il guardo o il bacio... e mirarvi la bocca e baciar gli occhi (11-12).

La gimcana semantica che queste collocazioni impongono costituisce un severo handicap per i musicisti; non sorprende, allora, trovare soltanto due partiture del madrigale nel Nuovo Vogel (mentre del Baciator dubbioso, con lo stesso tema, se ne registrano quindici).

Alle fonti già ricordate nel commento a 22 , va aggiunto il seguente madrigale del Guarini contenente, come questo, un'ottativa finale con cui si mira alla sinestesia:

Parole e baci

Con che soavità labbra odorate

e vi bacio e v'ascolto,

ma se godo un piacer, l'altro m'è tolto.

Come i vostri diletti

s'ancidono fra lor, se dolcemente

vive per ambiduo l'anima mia?

Che soave armonia

fareste o dolci baci, o cari detti

se foste unitamente

d'ambedue le dolcezze ambo capaci:

baciando i detti e ragionando i baci. (Rime 1598, n. 194) 
27 - Canz. 2 - Baci affettuosi ed iscambievoli. Aminta e Clori

28 - Canz. 3 - Baci dolci ed amorosi. Tirsi e Filli

27 - Canz. 2 - Baci affettuosi ed iscambievoli. Aminta e Clori

$1 \quad$ Poich'a baciar ne 'nvita

il sussurro del'onde

e quest'ombra romita

dal caldo sol n'asconde,

5 or ch'ardon fiori e foglie

e più le nostre voglie, bacinsi, o bella Clori, le nostre labra e nele labra i cori

Clori

Bacianne, Aminta mio,

10 io bacio se tu baci, bacia ch'io bacio anch'io: facciam, facciam di baci lunghe, lunghe catene, onde, dolce mio bene,

15 leghi e congiunga Amore seno a sen, labro a labro e core a core.

Aminta

Vita è del'alme il bacio e vita è di natura.

Mira, mentr'io ti bacio,

20 colà per la verdura:

non vedi come strette baciano i fior l'erbette?

bacian l'onde le rive?

bacian le fronde ancor l'aure lascive?

Clori

25 Dolce cosa è scontrarsi due bocche baciatrici.

Dolce cosa è baciarsi due liete alme felici.

Odi là nelo speco,

30 non senti tu com'Eco, mentr' un bacio s'imprime, invida del piacer mille n'esprime?

Aminta

Raddoppiam dunque i nodi 
cara mia Clori amata;

35 e se ' $n$ baciando godi, beatrice e beata, questo collo mi cingi ch'anch'io mentre mi stringi pareggiar ti prometto

40 quest'olmo qui, ch'ala sua vita è stretto.

Clori

Sieno i baci e gli amplessi, o sospirato Aminta, più profondi e più spessi, ch'io teco a prova avinta

45 giuro per quella face, ond'Amor mi disface, d'agguagliar con le braccia quell'edra là, che 'l caro tronco abbraccia.

28 - Canz. 3 - Baci dolci ed amorosi.Tirsi e Filli

Tirsi

1 Filli, cor del mio core, or che non è tra noi chi n'oda altri ch' Amore, dimmi, com'aver puoi

5 tanta dolcezza, oimé, ne'baci tuoi ?

Forse queste tue rose di rugiada son gravi? O fan l'api ingegnose nela tua bocca i favi?

10 Ond'è che baci dai tanto soavi?

S'Amor fuss'egli morto, la gioia incenerita e sepolto il conforto, la dolcezza infinita

15 poria d'un bacio tuo tornargli in vita.

Anzi, vita e dolcezza e ciò che si desia e ciò che più s'apprezza, baciata anima mia,

20 altro ch'un bacio tuo credo non sia.

Il dolce baciar tuo 
sì dolce il cor m'offende

ch' ei muor, ma 'l morir suo

l'aviva e più l'accende:

25 quel che morte gli dà, vita gli rende.

Tanto diletto io sento

mentre bacio e ribacio

che per farmi contento

apien, quando ti bacio,

30 trasformar mi vorrei tutto in un bacio.

Filli

Tirsi, vita ond'io moro, non già perch'io ti bei, ma sol perch'io t'adoro, sol perch' amante sei,

35 prendi tanto piacer de' baci miei.

11 vero mele ibleo, i) zucchero di canna, il balsamo sabeo, il nettare, la manna

40 è quel dolce desio che sì t'inganna.

Amor del bacio è fabro, egli il forma, ei lo scocca; pria dal cor che dal labro dolcemente trabocca,

45 ma "l sente e gode il cor più che la bocr $a$.

Amor che lega i cori lega i labri tenaci; di celeste licori intinge i nostri baci

50 temprandogli al' ardor dele sue faci.

Qualor con dolce rabbia bocca si bacia o morde, su le baciate labbia van con voglia concorde

55 a mordersi, a baciar l'anime ingorde.

Quando un molle rubino amante anima sugge, viene al'uscio vicino per fuggir, ma non fugge

60 che ' $n$ vita la sostien quel che la strugge. 
Tirsi

Baciami dunque, o Fille, d'amor dolce anelante; piovono i baci a mille, che baciato o baciante

6 per te sempre sarò felice amante.

\section{Filli}

Ecco ti bacio, o Tirsi, con bocca innamorata; corran l'alme ad unirsi, che baciante e baciata teco nel ciel d'Amor sarò beata.

Non solo la forma diversa distingue i due componimenti da quelli attigui: finite le schermaglie mordaci (23-24), caduta l'enfasi sulle sensazioni personali del maschio (25-26), la pace fatta e fruita con il libero esercizio del bacio si oppone anche al ritorno dell'inquietudine erotica dei Baci dolci amari (29) e della Guerra dei baci $(30)$ che porterà direttamente al riesplodere della passione nei conclusivi Amori notturni (31).

Nella seconda fase della vicenda osculatoria la doppia tappa narrativa di $\mathbf{2 7}$ e 28 presenta il momento di massima serenità, che costituisce dunque, se solo ai baci si vuole badare, un punto di arrivo. L'episodio è posto all'insegna della concordia: nulla turba la perfetta reciprocità dei baci, dalla richiesta all'accettazione, dalla riflessione teorica sulla natura dell'atto, condivisa dall'amante, all'invito a replicarlo, e infine alla nuova accettazione. Questo percorso è chiaramente scandito in $\mathbf{2 7}$, mentre in $\mathbf{2 8}$ l'azione si intende già avviata e l'uomo è colto inizialmente in estatiche interrogazioni sulla dolcezza del bacio; ad esse dà partecipe risposta la donna prima di ricevere il nuovo invito a congiungere le labbra raccolto nell'ultima stanza.

L'azione è affidata a due coppie diverse (Aminta e Clori, Tirsi e Filli) per ricordarci che qui non si ricostruisce un fatto reale, ma si dichiara l'esemplarità dei baci affettuosi ed iscambievoli, dolci e amorosi (e bastano i titoli ad avvertirci del rilievo assegnato alle combinazioni binarie); entrambe sono comunque di pastori per denotare la rustica, o pseudorustica, semplicità degli scambi amorosi.

Per quel che riguarda la struttura - una serie di strofe formate da un numero variabile di settenari seguiti da un endecasillabo - c'è innanzi tutto da riconoscere che essa è identica a quella di varie "ode" (e le altre di poco si discostano) del più volte ricordato Guido Casoni. Nullo risulta invece, sempre sul fronte metrico, un confronto con Chiabrera (cfr. Fasoli), già celebrato conzonettista all'altezza del 1602 (Canzoni 1586, 1588; Canzonette 1591; Scherzi e canzonette morali 1599; Le maniere dei versi toscani 1599). Ben dieci delle diciotto canzoni di $\mathbf{B}$ presentano questa struttura, con varianti da 3 a 
6 nel numero dei settenari, e da 16 a 210 in quello complessivo dei versi; e la stessa riapparirà in $\mathbf{C}$, in tre su dodici canzoni (con 5 o 7 settenari e con un numero di versi variabile da 132 a 240). La evidente semplicità dello schema della canzone, o canzonetta, o meglio ancora odicina (per segnare così la distanza rispetto alla canzonetta chiabreresca caratterizzata da una marcata scansione ritmica), non è legata a temi specifici. Troviamo infatti che qui come in $\mathrm{C}$ lo stesso schema è impiegato per argomenti vari che vanno dal pastorale al sacro.

Si tratta di un modulo lirico interessantissimo, a metà strada tra il disteso modello petrarchesco della canzone e il madrigale, ed è notevole che il poeta lo trovi a sé tanto congeniale da impiegarlo identico per anni senza farsi troppo tentare dalla sua estrema duttilità (dimostrata dal Casoni e dallo stesso Marino che in due casi aggiunge un secondo endecasillabo, in 138 e C Di 73, e in un altro ribalta l'ordine delle misure dei versi ( 8 endecasillabi e 1 settenario, in 144). Contribuiscono a caratterizzare ulteriormente questa forma mariniana la melicità del verso, le accumulazioni di elementi sintattici simili, le rime facili, la scioltezza del dettato e la diluizione dei concetti; che sono quindi, questi ultimi, distinti da quelli strutturalmente inattesi e sorprendenti dei madrigali. L'endecasillabo finale, di regola rimato con il verso precedente (nella stanza di canzone come nel madrigale), mostra qualche affinità con la chiusa del madrigale, dove infatti è il verso lungo che compare nella stragrande maggioranza dei casi: sebbene abbia perso la valenza epigrammatica serve comunque a siglare con una coda (grafica oltre che fonica) la conclusione del periodo e della stanza stessa. La netta distinzione sintattica è sfruttata sia in 27 , in cui per sei strofe si alternano le voci di Aminta e Clori, che in $\mathbf{2 8}$, in cui gli interventi più distesi degli amanti sono modulati da una serie di pensieri espressi individualmente nel giro della più breve strofa ( 4 versi rispetto ai 5 precedenti), e si concludono con lo scambio di una strofa per parte che vale strutturalmente quanto il distico conclusivo del madrigale.

Abbiamo sopra posto l'azione svolta nei due componimenti all'insegna della concordia; ora notiamo come questo fatto si traduca, nell'impiego del modulo binario, in una vistosa presenza dell' iterazione (a scapito dell'antitesi), proprio come indicano le due coppie di attributi nei titoli. C'è però una differenza notevole tra i due componimenti per quel che riguarda la presentazione del tema: nel primo è l'uomo che conduce l'azione e che si fa maestro dell'ars osculandi sentenziando: vita è del'alme il bacio / e vita è di natura (17-18); mentre nel secondo il controllo della situazione è affidato alla donna, che infatti enuncia la teoria: Amor del bacio è fabro / egli il forma, ei lo scocca... (41-45). 11 passo rivela un ricordo tassiano, Bacinsi insieme l'alme nostre anch 'elle: / fabro sia Amor che le distempri e sfaccia (Rime 380), e una somiglianza e contrario con un bacio di Filippo Alberti, che dal labro si parte e non dal core (Gareggiamento 64r), oltre ad annunciare una ripresa degli stessi versi nell'Adone: ...il cor nele mordaci / labra si bacia, amor del bacio è 
fabro, / il cor lo stilla, il labro poi lo scocca, / il più ne gode l'alma, il men la bocca (8.125).

Altre corrispondenze di qualche utilità si notano, in $\mathbf{2 7}$, nei seguenti passi: Vita è del'alme il bacio / e vita è di natura... (17-24); l'intera stanza sarà espansa nel canto di Talia dell'Adone (7.232-49), in cui troviamo, fra l'altro, Amor dunque sostegno è di natura (7.249). Erbe, fronde, aure e baci (23-25) sono coniugati insieme anche da Guido Casoni: ...aure feconde / che col lor dolce errore / fanno baciar le fronde / ... / e 'l matutino gelo, / che l'erbe imperla, $i$ baci son del cielo (Ode 24). Il bacio espressione dell'amore che informa la natura è anche cantato da Giovan Battista Leoni, Ecco baciansi $i$ cieli e gli elementi, / e lo stridor de' venti / son baci sonanti / da l'aria concitati / che fan l'erbe baciarsi per li prati / e ribaciar gli scogli i flutti amanti (Gareggiamento 46v, e si veda anche in $61 \mathrm{v}$ un altro madrigale dello stesso Leoni sullo stesso tema). Odi là nelo speco, / non senti tu com'Eco (29-30): nell'Adone, in circostanze narrative analoghe, riappariranno i medesimi termini in rima: Suonano i baci e mai dal cavo speco / forse a più dolce suon non rispos'Eco (8.146). L'immagine dell'olmo stretto alla vite (39-40) offre molti riscontri in Sannazaro, Tansillo, Tebaldeo, Tasso e Guarini; se ne riporta uno solo, del Tasso, che più degli altri l'ha usata: ...gli olmi mariti, a cui talor s'appoggia / la vite, e con piè torto al ciel se 'n poggia (Gerusalemme Liberata 3.75), ricordando anche le occorrenze del binomio nell'Adone (3.153, 4.47, $9.90,14.177)$. Non meno che la vite, l'edera abbraccia l'olmo o un generico tronco (47-49) da Serafino Aquilano (Rime, Ecl. 3:90) a Marino, qui come nell'Adone 4.177, 7.108, 7.246.

Per 28 un raffronto con 20 (70) è suggerito dal segmento 15-25. A Tirsi che all'altezza del verso 30 dice, trasformar mi vorrei tutto in un bacio, fa eco Francesco Contarini con la mia vita altro non è ch'un bacio (Gareggiamento $62 \mathrm{v}$ ). E infine i baci a mille (63) che richiamano il noto tópos catulliano, trovano riscontri in due basia di Giovanni Secondo ( $\mathrm{nn} .6,7$ ), in Rinaldi (Gareggiamento 62r) e in queste stesse Rime mariniane, poco più avanti in 31 (94) e nell'explicit di 50.

Resta da dire delle versioni in musica, 3 per 27 e ben 13 per 28 . La preferenza dei musicisti ancora una volta ci invita ad apporre qualche rilievo di ordine stilistico. L'andamento dialogico di 27, con l'alternarsi pur regolare delle voci diverse di Aminta e Clori, impone la replica della conversazione sullo spartito, il che naturalmente già costituisce un limite alla libertà espressiva dei musicisti. Ma le differenze più vistose si registrano nella misura della stanza $\mathrm{e}$ nell'uso della rima. Più breve, e quindi più cantabile (cinque versi rispetto a otto), la strofa di 28 presenta più ricorrenze eufoniche, non solo perché contiene un numero minore di rime (due rispetto a quattro), ma perché spesso vi compaiono rime vocaliche; se ne contano cinque (in tredici versi) tra le ventotto rime di $\mathbf{2 8}$, mentre una sola (in due versi) compare tra le ventiquattro di 27. Si deve registrare infine l'ovvia difficoltà di traduzione in musica della 
seconda stanza di $\mathbf{2 7}$, quasi un madrigale a sé stante, in cui si affollano incalzanti iterazioni (bacio - baci, bacia - bacio, io - io, facciam - facciam, lunghe - lunghe) concluse esplosivamente nell'endecasillabo finale (seno a sen, labro a labro e core a core) e quindi con una espressività già fortemente definita: le ripetizioni, che per il poeta costituiscono qui l'artificio di maggior rilievo, sono invece comunissime, se non addirittura inevitabili, nel canto (e in particolare in quello a più voci) che perciò tende a vanificare la peculiare attrattiva di un brano poetico di questo tipo.

29 - Mad. 26 - Baci dolci amari

30 - Mad. 27 - Guerra di baci

29 - Mad. 26 - Baci dolci amari

1 Soavissimi baci, baci non già, ma strali, dolci sì, ma mortali: in voi temprar l'incendio ebbi speranza,

5 ma più cresce e s'avanza e là dove d'Amor l'ambrosia provo ivi il tosco ritrovo. Tal sitibondo infermo ricorre a le dolci acque e mentre beve

10 dal refrigerio suo morte riceve.

30 - Mad. 27 - Guerra di baci

$1 \quad$ Feritevi, ferite viperette mordaci, dolci guerrere ardite, del diletto e d'Amor bocche sagaci!

5 Saettatevi pur, vibrate ardenti l'arme vostre pungenti!

Ma le morti sien vite, ma le guerre sien paci, sien saette le lingue e piaghe i baci.

Ancora una volta, e sarà l'ultima, è con una coppia di componimenti che ci viene presentato un segmento della vicenda osculatoria. Ma la binarietà che in 27 e 28 si era posta all'insegna della concordia acquista qui tutt'altra valenza: si deve infatti parlare di inquietudine e di tensione tra opposte spinte emotive. La dispositio ci esprime con inequivocabile chiarezza che la situazione rappresentata nei due madrigali si è ora caricata di un erotismo più intenso del prece- 
dente e che ci stiamo avvicinando al rapporto sessuale completo dei conclusivi Amori notturni. Spie vistose della mutata atmosfera sono i due ossimori (nel titolo di 29 e al v. 3 di 30) e la serie nutrita di antitesi (in 29 nei vv. 2, 3, 4-5, 67,10 ; in 30 nei vv. $7,8,9$ ) che vengono a opporsi alle iterazioni precedenti denotanti una più serena soddisfazione nell'esercizio del bacio. Le metafore si fanno dure, guerresche, perfino violente, e indicano uno sconvolgimento generale dei sensi: saette e lingue si intrecciano e si confondono con dolcezze e amarezze, con ambrosia e tosco, guerre e paci, morti e vite. Ansia e desiderio, in crescendo fino alla chiusa di $\mathbf{3 0}$, proiettano l'azione in avanti in un turbine di eccitazione che tende a illustrare il massimo dell'erotismo fomentato dai baci (che infatti, vedremo, in $\mathbf{3 1}$ cederanno il posto al nuovo e ultimo culmine erotico costituito dal rapporto sessuale vero e proprio).

Riscontri testuali notevoli, oltre ai più ovvi con la precedente canzone dei Baci e con le sue segnalate fonti, sono ancora con altre opere mariniane. Il rarissimo vezzeggiativo di "vipera", e per di più in combinazione con "saetta", ricompare nell'Adone, in cui saetta e scocca / viperetta amorosa (8.122); ma si deve ricordare che la lingua baciatrice è per Francesco Scaglia un umidetto e mobil serpe che avvelena e morde (Gareggiamento 60r). Ambrosia e tosco, con i termini "nettare" e "veleno", compaiono nella Galeria (n. 8.7-8: 139) e nell'Adone (1.162).

Nel Nuovo Vogel risultano sette partiture di 29 e diciassette di 30 . Per spiegare la differenza si notino innanzi tutto, nel primo madrigale, le due rime irrelate $(a, e)$ e poi il numero maggiore di versi e di endecasillabi. La sintassi è più piana nella Guerra di baci e il livello di espressività, per i vocativi dell'incipit e le ottative finali, certamente più elevato.

\section{1 - Canz. 4 - Amori notturni}

1 Quando stanco dal corso a Teti in seno per trovar posa e pace

Febo si corca e "I di ne fura e cela, e nel tranquillo mar, nel ciel sereno

5 ogni Euro, ogni aura tace, dorme il marino armento e l'onda gela, allor ch'emula al giorno, notte spiegando intorno il suo manto gemmato il mondo vela,

10 e tant'occhi apre il ciel quanti ne serra, vaghi di sonno e di riposo in terra;

allor Lilla gentil, l'anima mia, dala gelosa madre e dal ritroso genitor s'invola; 
15 indi per chiusa e solitaria via,

di vaghe orme leggiadre

stampa l'arena, e taciturna e sola

(se non quanto va seco

Amor per l'aer cieco),

20 mentre pesce non guizza, augel non vola, rinchiusa in un beato antro $m$ 'attende, antro che dale fate il nome prende.

Io, cui lunge da lei grave è la vita, tosto che ' 1 ciel s'inbruna,

25 sconosciuto colà drizzo le piante.

Quasi notturno sol, la via m'addita

nuda e senz'ombra alcuna

Cinzia, qual pria s'offerse al caro amante, e già ferir la miro

30 dal argentato giro

di ceruleo splendor l'onda tremante;

e fatte a mio favor più che mai belle

spettatrici d'amor veggio le stelle.

Giunto al mio ben, chi poria dir gli spessi,

35 i lunghi, i molli baci?

i sospir tronchi? i languidi lamenti?

Chi può contar degli amorosi amplessi

le catene tenaci?

gli accesi sguardi? gl'interrotti accenti?

$40 \quad$ gli atti dolci e furtivi?

gli atti dolci e lascivi?

Tanti sono i diletti e sì possenti

che dal cor per se stessa si divide

l'anima, e innanzi tempo amor m'uccide.

45 Lentando allor, ma non sciogliendo il laccio, con la prima dolcezza

temprato alquanto il fervido desio, languidamente l'un al'altro in braccio vinti dala stanchezza

50 ce ne stiam vaneggiando ed ella ed io.

Mentr'io pian pian col manco

a lei stringo il bel fianco

e con l'altro altra parte ascosa spio, ella d'ambe le sue, peso non grave,

55 fa quasi al collo mio giogo soave.

Io narro a lei favoleggiando intanto, quando primier mi prese e l'ora e "l punto e la maniera e '1 loco; 
poi dico: - E da quel dì ch'Amor cotanto

60 degli occhi tuoi m'accese, sprezzai, si dolce n'arsi, ogni altro foco.

Questi il mio 'ncendio furo

e per questi ti giuro

che d'ogni altra bellezza mi cal poco.

65 Crocale il ti può dir, Crocale figlia

d'Alceo, bench'ella bruna e tu vermiglia.

Questa ognor mi lusinga e prega e chiama, ma tutto indarno. - Allora

mi risponde colei ch'io stringo e suggo:

70 - Caro Fileno, e tu non sai se m'ama

e mi segue e m'adora

Tirinto il biondo, e s'io l'aborro e fuggo?

Quanti doni mi porge, misero, e non s'accorge

75 ch'io per te solo... - e vuol seguir "mi struggo",

ma mosso dal piacer che "l cor mi tocca

le chiudo allor la sua con la mia bocca.

Qui risorto il desio, qual d'arco strale, ver l'ultimo diletto

80 sen corre a sciolto fren carco d'ardore.

Tra noi scherzando e dibattendo l'ale,

l'ignudo pargoletto

fa traboccar d'estrema gioia il core.

Sul'arena a cadere

85 n'andiam: con qual piacere,

questo mi tacerò, dicalo Amore;

anzi faccial per prova altrui sentire,

ché forse anch'egli, Amor, nol sapria dire.

Stanco, non sazio, al fine alzo a' begli occhi

90 gli occhi tremanti e poi

da le sue labra il fior del'alma coglio;

e mentre il molle seno avien ch'io tocchi

e vo' tra' pomi suoi

scherzando e mille baci or dono or toglio

95 tal, che lasso pareva,

pronto si desta e leva,

ond'io pur di morir dolce m'invoglio;

ma là dove più ingordo altri si sforza,

per soverchio desir manca la forza.

100 Così mi giaccio inutil pondo appresso

ala mia ninfa amata 
che mi deride stupido ed insano.

Perch'io m'adiro e dico: - $\mathrm{O}$ di me stesso

parte vile insensata

105 chi più giamai t'aviverà, se 'nvano

si vezzosa ed amica

più volte s'affatica

di farti risentir la bella mano?

Certo di sasso sei; ma come, ahi lasso!

110 come sì molle sei, se sei di sasso? -

Ed ecco uscir fuor dele rive estreme

del indica pendice

rapido il sol dala sua nunzia scorto.

Ella, ch'esser veduta ha scorno e teme,

115 sospirando mi dice:

- A Dio, ben rivedrenne e fia di corto,

a che tanto affannarte? -

Poi mi bacia e si parte;

lo resto e dico: - Invan per me se' sorto,

120 invido sol, che questa notte oscura

era a me più che "l dì lucida e pura.-

Canzon notturna sei,

notturni i furti miei;

non uscir, prego, al sol, fuggi la luce:

125 oblio più tosto eterno, ombra profonda

le mie vergogne e i tuoi difetti asconda.

Con un impianto decisamente narrativo, quasi una sigla della priorità delle funzioni del racconto finora esplicitate dalla pur lucida dispositio, questi Amori notturni chiudono la seconda tornata e l'intero ciclo dei baci. L'evento riportato è un incontro di Fileno e Lilla, amanti che dall'onomastica mariniana riconosciamo come il poeta e la sua donna.

Anche qui, come nella canzone dei Baci, cui per collocazione, tema e struttura conviene rifarsi, l'amplesso rappresenta l'approdo ultimo delle più tenere effusioni, ma la progressione erotica è ora accelerata e i baci sono solo brevemente menzionati $(34-35,76-77,91,118)$ per lasciar spazio ai tre rapporti sessuali che scandiscono l'azione e i tempi dell'incontro notturno. Va subito precisato, prima di analizzare più da vicino il componimento cosi ripartito, che il terzo amplesso non riesce (per soverchio desir manca la forza, 99): un fallimento che simbolicamente riafferma la coriacea fissità del doppio, ovvero dell'onnipresente legge del due. E che questa poi apra ideologicamente a un'insanabile malinconia, con innumerevoli esempi di "fallimento dell'incontro sessuale come mezzo di umana intesa ed uguaglianza" (Pozzi 6-7) è cosa da tenere ben presente per evitare il rischio di ricollocare questo e altri componimenti affini nel lazzaretto delle miserie oscene. 
Nel lento giro sintattico con cui si dà inizio all'azione introducendo, con tre proposizioni temporali, Lilla gentil che si reca all'appuntamento, è notevole il rilievo assegnato alla coppia "cielo e mare" espresso da un doppio chiasmo: tranquillo mar - ciel sereno, 4; dorme il marino armento - l'onda gela, 6. Ai due elementi non è associata la "terra", come avverrà invece in un luogo dell'Adone che riporta gli stessi sintagmi, sereno il ciel... tranquillo il mar... verdeggiante la terra $(20,8-10)$, per cui si può pensare a una volontà di esprimere l'intimo isolamento del luogo dell'incontro: una marina alcova astratta dal resto del mondo come un'aperta conchiglia sulla sabbia. Gli schermi di protezione sono di nuovo evocati due altre volte più in basso: mentre pesce non guizza, augel non vola (20); Cinzia... ferir miro... dal'argentato giro l'onda tremante (28-31).

Non due come a Lilla, ma una sola stanza è sufficiente a Fileno per giungere alla grotta delle sirene allettato com'è, Endimione marittimo, dall'immagine antropomorfa della luna, nuda e senz'ombra alcuna / ...qual pria s'offerse al caro amante (27-28).

Una serie concitata di interrogazioni retoriche presenta i preliminari dell'amplesso che, nonostante includa baci lunghi, oltre che spessi e molli, lo stile vuole rapido. Gli ultimi tre versi della stanza che lo descrivono (42-44), con tanto di eiaculazione precoce, vanno rapportati ai sei del meno affrettato secondo rapporto sessuale (78-83). Maestro di sottili ambiguità, qui il Marino non sta certo porgendoci un messaggio su cui sia possibile equivocare, ma è notevole che ad esse egli ricorra evitando la gravezza di precise denotazioni. Il gioco d'equilibrio continua nell'area della terza prova sessuale, quando l'uso comune (nella poesia più lasciva o burlesca) del termine esatto per indicare il pene, "cotale", viene ridotto a tal (95) che quasi si nasconde dopo i più netti e chiari occhi, labra, seno, pomi e baci (88-94).

La pausa che segue il primo amplesso è aperta da un verso, lentando allor, ma non sciogliendo il laccio (45), che è il primo segno della fondamentale cieca insistenza nell'atto liberatorio del sesso. Più disforicamente scoperto, il rilievo ritornerà puntuale dopo il secondo amplesso, stanco non sazio... (89), quando anticiperà la descrizione conclusiva dell'angosciosa condizione dell'io narrante. Osserviamo che mentre la canonica irraggiungibilità della donna è ormai un fatto poetico superato, l'euforia dei baci corrisposti e dell'unione sessuale ha fatto insorgere una ansietà nuova che non sarà azzardato definire già moderna ed esistenziale.

Assume allora un significato parodico il fruitissimo sintagma petrarchesco, l'ora e 'l punto, 58 (Rerum Vulgarium Fragmenta 61.2; Poliziano 107.15 e 109.1; Lorenzo 63.37; Filenio Gallo 1.127.95 e 4.13.2; Boiardo 147.1; Firenzuola 36.3; Bandello 102.83) ripreso anche nell'Adone (2.164), ed eversiva sarà anche la chioma, non più bionda ma rossa, dell'amata (66). Non meno eccentrica risulterà la menzione di Crocale (62-66) e dei corteggiamenti di Tirinto il biondo (70-75), la cui funzione narrativa è di portare in campo il 
motivo della gelosia per riaccendere il desiderio sessuale.

L'ultimo diletto (79) che accenna alla descrizione dell'amplesso nei Baci (vinta allor dal diletto / con un sospir sen viene / l'anima al varco e il proprio albergo oblia, 85-87), è accompagnato dall'immagine dello strale amoroso che, in circostanze analoghe, ricomparirà nell'Adone (drizzar si sente al cor l'acuto strale, 8.65). Il core infine trabocca (83), ma in maniera più temperata, in Tasso (Rime 868).

Nella stanza che segue spiccano per inefficacia, pallidi esempi dei molti altri precedenti più vivaci, i mille baci $(94)$ che, nonostante l'ausilio di profonde carezze (92-93), non conducono al nuovo desiderato morir dolce (97).

La bella mano (108) che invano più volte s'affatica (107) è un'altra vistosa parodia che dai Rerum Vulgarium Fragmenta (37.16 e 200.1) al canzoniere di Giusto de' Conti, dalle Rime del Bembo a quelle del Tasso, investe uno dei luoghi topici più rappresentativi dell'intera tradizione lirica in volgare. L'antitesi che fa seguito al noto sintagma (109-10) si presenta con una comicità a dir poco problematica, non solo per l'immagine sconcia del membro flaccido su cui è basata, ma per il ben più stilisticamente intrigante artificio adrigalesco. $\mathrm{Ci}$ sembra che, in un componimento dai versi controllatissimi come questo, l'abbandono burlesco dell'intera stanza, esasperato in fondo dal rincorrersi delle paronomasie sul registro serpeggiante della sibilante (sa- -so se- -so / si sese se- sa- -so), non si spieghi se non con una precisa volontà di dissacrazione. La canzone è del resto notturna, come ci ricordano l'ultima stanza e il congedo, in quanto offre un'alternativa privatissima e luminosa, più che 'l dì lucida e pura (121), alla solare e ordinata, ma blanda, realtà del senso poetico comune.

Non s'è detto della musica, ma si sarà già intuita l'intraducibilità della canzone sul pentagramma: il Nuovo Vogel non segnala alcuna partitura.

\section{University of Toronto}

\section{NOTE}

1 I numeri in grassetto, senza altra indicazione, si riferiscono ai componimenti della Lira II. I testi mariniani si riproducono dall'edizione delle Rime del 1602. Per la trascrizione mi attengo ai criteri adottati da Ottavio Besomi e Alessandro Martini nell'edizione delle Rime amorose. Da questa stessa edizione, per abbreviazioni e sigle riferite all'intera Lira (Rime I, Rime II, Lira III), traggo lo schema seguente:

Parti Sezioni Componimenti

Rime. Parte prima. Venezia 1602

$\mathbf{A}$

Amorose

Am

Marittime

Ma

1-50

Boscherecce

Bo

1-88

Eroiche

Er 1-64

Lugubri

Lu 


\begin{tabular}{|c|c|c|c|}
\hline Morali & & Mo & $1-16$ \\
\hline Sacre & $y_{0}$ & Sa & $1-41$ \\
\hline Varie & & $\mathrm{Va}$ & $1-17$ \\
\hline Proposte Marino & & $\operatorname{Pr}$ & $1-11$ \\
\hline Proposte Altri & & Pra & $1-24$ \\
\hline Risposte Marino & & $\mathbf{R i}$ & $1-24$ \\
\hline Risposte Altri & & Ria & $1-11$ \\
\hline Rime. Parte seconda. Venezia I 602 & B & & $1-225$ \\
\hline Lira.. Parte terza. Venezia I 614 & C & & \\
\hline Amori & & Am & $1-136$ \\
\hline Lodi & & Lo & $1-86$ \\
\hline Lagrime & & $\mathbf{L a}$ & $1-40$ \\
\hline Divozioni & & Di & $1-87$ \\
\hline Capricci & & $\mathrm{Ca}$ & $1-64$ \\
\hline
\end{tabular}

Avverto, inoltre, che per le poesie di Marino segno il numero dei versi tra parentesi dopo la citazione. Per evitare confusione, visto il gran numero di riferimenti necessari al commento dei testi, ricorro quando è d'uopo a 'prosaiche' indicazioni di pagina (i.e. "p. xx") o di numero di

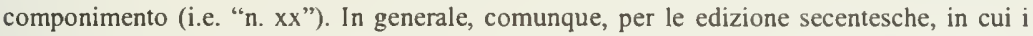
componimenti non sono numerati, le cifre tra parentesi segnano la pagina, mentre per le edizioni moderne segnano il componimento. Indico i loci del Gareggiamento con il numero di carta, mentre quelli dell'Adone, ovviamente, con quelli di canto e ottava.

2 I madrigali osculatorî del Gareggiamento sono raccolti sotto queste intestazioni: Bocca ritrosa, Baci desiderati, Bacio chiesto, Baci semplici, Bacio mordace, Bacio involato, Baci dolci, Un bacio è poco, Bacio ricevuto, Baci reciprochi, Fanciul baciato, Bocca morsicata, Fiori in bocca. Tutti si trovano nella Parte seconda, intitolata Le Dipendenze, Orero Madrigali Amorosi (Ir-128r). Le carte sono numerate separatamente per la Prima e la Seconda parte, le più lunghe; dalla Terza alla Nona, l'ultima, la numerazione è progressiva. Gli esempi che di seguito si riportano sono tutti tratti dalla Seconda parte. Per un'ampia descrizione di questo fondamentale testo di riferimento rimando all'articolo di Alessandro Martini, "Ritratto del madrigale poetico fra cinque e seicento".

3 Si tratta del componimento n. 277 nell'edizione di lavoro di Antonio Vassalli. Ho ritoccato l'interpunzione e dato la maiuscola ad "Amore", Cupido. Dalla collocazione assegnata dal Vassalli a questa canzone si intende che essa proviene da una stampa miscellanea che precede le Rime del '98. La prossima, annunciata, edizione chiarirà ogni dubbio.

4 Segnalato dal Kristeller nel suo Iter Italicum, il ms. si trova nella Thomas Fisher Rare Book Library della University of Toronto e reca la seguente catalogazione: "RBSC, Stillman Drake Galileo Collection, v. 5 - RBms 88.066". Contiene 257 lettere di Girolamo Magagnati e un capitolo da lui composto all'indirizzo del Rinuccini (Penso si fisso che mi vien l'accidia [manca la numerazione di carta, o pagina, ma pp. 190-99]). Il ms. misura mm. $180 \times 260$. La legatura è secentesca. Le pagine sono numerate con eccezione delle ultime che contengono i versi. Il carattere calligrafico e la pressoché totale assenza di cancellature fanno pensare a una copia "in pulito" destinata a una stampa, mai avvenuta, oppure a una silloge preparata in omaggio a un personaggio in vista, ovvero al Giulio Melchiori, destinatario di alcune lettere della raccolta, menzionato nel frontespizio. Che è il seguente: "LETTERE / DEL SIG: GIROLAMO / MAGAGNATI / a diversi / dedicate / AL MOLTO ILL: SIG: / IL SIG: GIULIO MELCHIORI". Mancano indicazioni di luogo e data, ma a p.3 troviamo la lettera di dedica del curatore, "Rocho Agud:", "Di Venetia li x Gen . 1620". Seguono le 257 lettere (e, in fondo, il capitolo al Rinuccini) da cui sono stati espunti i luoghi di provenienza e le date perché, evidentemente, il curatore non si poneva fini documentari bensì voleva mostrare le doti letterarie del Magagnati: dice infatti le sue lettere "pregne di concetti, piene di vaghezze, belle nella tessitura, ordinate alla regola e 
terse nella lingua" (3) senza spendere una sola parola sull'importanza dei destinatari. Tra i quali è appunto il Marino (sette lettere) insieme a Galilei, Guarini, Chiabrera, Rinuccini, Angelo Grillo, Claudio Achillini, Alessandro Striggio e altri. Conto di dar presto un dettagliato resoconto di questo notevole manoscritto e, in particolare, della parte che riguarda il Cavaliere napoletano.

\section{OPERE CITATE}

Alberti, Filippo. /l Gareggiamento, passim.

Aquilano, Serafino. Vedi Ciminelli.

Ariosto, Ludovico. "Le Rime". Opere minori. Ed. Cesare Segre. Milano-Napoli: Ricciardi, 1954. 107-237.

Bandello, Matteo. Rime. Ed. Massimo Danzi. Modena: Panini, 1989.

Belli, Valerio. Il Gareggiamento, passim.

Bembo, Pietro. "Le Rime". Prose e rime. Ed. Carlo Dionisotti. Torino: UTET, 1966. 505-687.

Bianconi, Lorenzo. "Poesia e musica. Il Cinquecento e il Seicento". Letteratura italiana. Ed. Alberto

Asor Rosa. Vol. 6. Teatro, musica, tradizione dei classici. Torino: Einaudi, 1986. 319-63.

Boiardo, Matteo Maria. Amorum Libri III. Opere volgari. Ed. Pier Vincenzo Mengaldo. Bari:

Laterza, 1962. 1-128.

Borzelli, Angelo. Il Cavalier Giambattista Marino. Napoli: Priore, 1898.

Casoni, Guido. Il Gareggiamento, passim.

Ode del S. Guido Casone. Accresciute e distinte in tre parti. Treviso: Appresso Angelo Reghettini, 1615 (Prima ediz. 1602).

Chiabrera, Gabriello. Delle opere di G. C. in questa ultima impressione tutte in un corpo novellamente unite. Venezia: Baglioni, 1805.

Ciminelli, Serafino de' [Serafino Aquilano]. Die Strambotti des Serafino Dall'Aquila. Ed. Barbara Bauer-Formiconi. München: Wilhelm Fink Verlag, 1967.

Le rime. Ed. Mario Menghini. Bologna: Romagnoli Dall'Acqua, 1894.

Colonna, Vittoria. Rime. Ed. Alan Bullock. Roma-Bari: Laterza, 1982.

Contarini, Francesco. Il Gareggiamento, passim.

Fasoli, Paolo. "Non prima ebbe favella che vena': Preliminari per un discorso su Marino e

Chiabrera". The Sense of Marino: Literature, Fine Arts and Music of the Italian Baroque. Ed.

Francesco Guardiani. New York-Ottawa-Toronto: Legas, 1994. 345-360.

Fiamma, Carlo. Il Gareggiamento, passim.

Firenzuola, Agnolo. "Rime". Opere. Ed. Adriano Seroni. Firenze: Sansoni, 1958. 773-1024.

Gallo, Filenio. Rime. Ed. Maria Antonietta Grignani. Firenze: Olschki, 1973.

Il Gareggiamento. Il Gareggiamento poetico etc.

Guarini, Battista. Il pastor fido. Ed. Ettore Bonora. Commento di Luigi Banfi. Milano: Mursia, 1977.

II Gareggiamento, passim.

Poesie. Edizione di lavoro fornita da Antonio Vassalli comprendente, oltre alle Rime del 1598 (Venezia: Ciotti), componimenti tratti da numerose raccolte miscellanee.

Guizzardi, Gioan Maria.ll Gareggiamento, passim.

Il Gareggiamento poetico del Confuso Accademico Ordito [i.e. Carlo Fiamma]. Madrigali amorosi, gravi e piacevoli ne quali si vede il bello, il leggiadro e il vivace dei più illustri poeti d'Italia.

9 parti. Venezia: Barezzo Barezzi, 1611.

Leoni, Giovan Battista. Il Gareggiamento, passim.

Magagnati, Girolamo. Lettere. Manoscritto della Thomas Fisher Rare Book Library, University of Toronto. Senza segnatura.

Magno, Celio. Rime di Celio Magno et Orsatto Giustiniano. Venezia: A. Muschio, 1600. Per la numerazione delle rime ho fatto riferimento all' edizione di lavoro fornita da Francesco Erspamer. Marino, Giovan Battista. L'Adone. Ed. Giovanni Pozzi. 2 tomi. Milano: Adelphi, 1988. La Galeria. Ed. Marzio Pieri. Padova: Liviana, 1979. 
- La Lira. Terza parte. Venezia: Ciotti, 1614.

. La Sampogna. Ed. Vania de Maldé. Milano-Parma: Fondazione Pietro Bembo /

Ugo Guanda Editore, 1993.

. Rime. Prima e seconda parte. Venezia: Ciotti, 1602.

Rime amorose. Ed. Ottavio Besomi e Alessandro Martini. Modena: Panini, 1987.

Martini, Alessandro. "Marino postpetrarchista". Versants 7 (1985): 15-36.

$529-48$.

"Ritratto del madrigale poetico fra Cinque e Seicento". Lettere italiane 33 (1981):

Medici, Lorenzo de'. "Canzoniere”. Tutte le opere. Ed. Paolo Orvieto. Roma: Salerno, 1992.

Moro, Maurizio [M.M.]. Il Gareggiamento, passim.

Murtola, Gasparo. Il Gareggiamento, passim.

Nardi, Agostino. Il Gareggiamento, passim.

Nuovo Vogel = Vogel, Emil et al. Bibliografia della musica italiana vocale profana: pubblicata dal 1500 al 1700. Nuova edizione interamente rifatta e aumentata con gli indici dei musicisti, poeti, cantanti, dedicatari e dei capoversi dei testi letterari. Pomezia : Staderini-Minkoff, 1977.

Perella, Nicolas James. The Kiss Sacred and Profane: An Interpretative History of Kiss Symbolism and Related Religio-erotic Themes. Berkeley: U of California P, 1969.

Petracci, Pietro. Il Gareggiamento, passim.

Petrarca, Francesco. Canzoniere. Ed. Gianfranco Contini. Torino: Einaudi, 1964.

Poliziano, Angelo. Rime. Ed. Daniela Delcomo Branca. Firenze: Accademia della Crusca, 1986.

Pozzi, Giovanni. Introduzione e Commento a Giovan Battista Marino. L'Adone. Tomo 2.

Ragarin, Alessandro. Il Gareggiamento, passim.

Rinaldi, Cesare. Canzoniere. Bologna: Eredi di Gio. Rossi, 1601.

Delle rime. Parte terza. Bologna: Benacci, 1590.

Delle rime. Parte sesta. Bologna: Benacci, 1598.

.ll Gareggiamento, passim.

Scaglia, Francesco. Il Gareggiamento, passim.

Secondo, Giovanni. Vedi Secundus.

Secundus, Joannes. "Basia". The Love Poems of Joannes Secundus. A Revised Latin Text and an English Verse Translation. Ed. Frederick Adam Wright. London: George Routledge, 1930.

Simon, Roger. "I sospiri, Chanson oubliée de Giovan Battista Marino". Revue des études italiennes 108 (1972): 61-73.

Stigliani, Tommaso. Rime. Venezia: Ciotti, 1601.

Taddeo, Edoardo. "Di una non del tutto dimenticata canzone di Giovan Battista Marino". Giornale Storico della Letteratura Italiana 151(1974): 586-90.

Tansillo, Luigi. Canzoniere. Ed. Erasmo Percopo. Napoli: Tipografia degli Artigianelli, 1926.

Tarsia, Galeazzo di. Rime. Ed. Cesare Bozzetti. Milano: Fondazione Arnoldo e Alberto Mondadori, 1980.

Tasso, Torquato. Gerusalemme liberata. Ed. Lanfranco Caretti. Milano: Mondadori, 1988. .Il Gareggiamento, passim.

"Rime". Opere. Ed. Bruno Maier. Milano: Rizzoli, 1963. 1.209-1186; 2.1-439.

Verità, Marco. Il Gareggiamento, passim. 